\title{
Level set topology and shape optimization by density methods using cut elements with length scale control
}

\author{
Andreasen, Casper Schousboe; Elingaard, Martin Ohrt; Aage, Niels
}

Published in:

Structural and Multidisciplinary Optimization

Link to article, DOI:

$10.1007 / \mathrm{s} 00158-020-02527-1$

Publication date:

2020

Document Version

Peer reviewed version

Link back to DTU Orbit

Citation (APA):

Andreasen, C. S., Elingaard, M. O., \& Aage, N. (2020). Level set topology and shape optimization by density methods using cut elements with length scale control. Structural and Multidisciplinary Optimization, 62, 685-707. https://doi.org/10.1007/s00158-020-02527-1

\section{General rights}

Copyright and moral rights for the publications made accessible in the public portal are retained by the authors and/or other copyright owners and it is a condition of accessing publications that users recognise and abide by the legal requirements associated with these rights.

- Users may download and print one copy of any publication from the public portal for the purpose of private study or research.

- You may not further distribute the material or use it for any profit-making activity or commercial gain

- You may freely distribute the URL identifying the publication in the public portal 


\title{
Level set topology and shape optimization by density methods using cut elements with length scale control
}

\author{
Casper Schousboe Andreasen - Martin Ohrt Elingaard - Niels Aage
}

Received: date / Accepted: date

\begin{abstract}
The level set and density methods for topology optimization are often perceived as two very different approaches. This has to some extent led to two competing research directions working in parallel with only little overlap and knowledge exchange. In this paper we conjecture that this is a misconception and that the overlap and similarities are far greater than the differences. To verify this claim, we employ, without significant modifications, many of the base ingredients from the density method to construct a crisp interface level set optimization approach using a simple cut element method. That is, we use the same design field representation, the same projection filters, the same optimizer and the same so-called robust approach as used in density based optimization for length-scale control. The only noticeable difference lies in the finite element and sensitivity analysis, here based on a cut element method, which provides an accurate tool to model arbitrary, crisp interfaces on a structured mesh based on the thresholding of a level set - or density - field. The presented work includes a heuristic hole generation scheme and we demonstrate the design approach on several numerical examples covering compliance minimization and a compliant force inverter. Finally, we provide our Matlab code, downloadable from www.topopt.dtu.dk, to facilitate further extension of the proposed method to e.g. multiphysics problems.
\end{abstract}

The authors thank the Villum Foundation for support through the Villum Investigator project InnoTop.

Casper Schousboe Andreasen

Department of Mechanical Engineering, Section for Solid Mechanics, Technical University of Denmark, Nils Koppels Allé, Building 404, DK-2800 Kgs. Lyngby

Tel.: +45 45254262

E-mail: csan@mek.dtu.dk
Keywords Topology optimization - Cut elements · Level set methods · Density methods · Length scale control

\section{Introduction}

Since its introduction in the late 1980's (Bendsøe and Kikuchi 1988) the material distribution method, known as topology optimization, has shifted from an academic research discipline to an important and commonly used tool in many industries. In engineering it is used for the design of structural components e.g. in aerospace and automotive industries (Bendsøe and Sigmund 2004), while many architects and industrial designers use it as inspiration (Beghini et al. 2014). The success of the design methodology has led to its application in several other physical disciplines such as fluid dynamics (Borrvall and Petersson 2003; Andreasen et al. 2009; Dilgen et al. 2018), acoustics (Dühring et al. 2008; Christiansen and Sigmund 2016), MEMS devices (Larsen et al. 1997; Sigmund 2001b), large-scale and giga-resolution design (Evgrafov et al. 2008; Aage et al. 2017) and even as interactive educational tools (Aage et al. 2013; NobelJørgensen et al. 2016). As a consequence of the vast popularity of the design method, a large number of variants exist in the literature, of which, the two dominant ones are the density based (Bendsøe 1989; Zhou and Rozvany 1991; Wang et al. 2011; Sigmund and Maute 2013) and the level set based approaches (Osher and Sethian 1988; Sethian and Wiegmann 2000; Wang et al. 2003; Van Dijk et al. 2013). Within each of the two major approaches there exist a multitude of different directions. For example, the term level set topology optimization is used for methods that employ an ersatz material model c.f. Fig 1(c) (Allaire et al. 
2004; Wang and Wang 2006); methods that use extensive remeshing and exact boundary tracking, c.f. Fig. 1(b) (Allaire et al. 2013); methods that perform the design update using mathematical programming (Van Miegroet and Duysinx 2007; Kreissl and Maute 2012) and those that update the design by time-stepping an advection-diffusion type equation (Allaire et al. 2004; Chen et al. 2008). For density methods, both nodal and element-wise design representations are employed and there exist several approaches to performing the design update, e.g. BESO (Querin et al. 1998) and mathematical programming. As a result, the number of variations are numerous, and mentioning all is outside the scope of this paper. Despite their different mathematical formulations and numerical implementations, we argue that they share more similarities than differences (as also pointed out in e.g. Sigmund and Maute (2013)). The main similarity, and a common trade for all successful methods, both level set and density based, is that they are based on sensitivity information (Sigmund 2011). In this work we focus solely on the transition from a density representation to a level set based formulation, and show that this only requires an update of the underlying finite element analysis and minor modification to the sensitivity computations. All other aspects remain exactly as those used in density methods.

Depending on the physics at hand, the need for an explicit boundary representation may be crucial for the successful application of structural optimization. For example, the works (Sigmund and Clausen 2007; Yoon et al. 2007; Lundgaard et al. 2018) present density based methods in which the interface is of primary importance and all illustrate the difficulties in introducing the appropriate interface conditions using a density representation. Therefore, methods capable of an accurate representation of such conditions are of high interest to the community. To realize the accurate modelling of a crisp boundary we employ a cut element method. The proposed method can, in general terms, be viewed as a subset of XFEM (Daux et al. 2000; Belytschko et al. 2003), CutFEM (Hansbo and Hansbo 2002; Burman et al. 2014) or the Finite Cell method (Düster et al. 2008) if no enrichments or stabilization is used, and if the entire background domain is modelled at all times. In other words, the proposed cut element approach allows for a crisp boundary representation while working on a fixed structured background mesh with constant degree of freedom (dof) numbering, c.f. Fig. 1(d). As the proposed cut element approach does not require the introduction of any additional enrichment dofs, it is well suited for large-scale topology optimization in a parallel environment, since the domain decomposition and dof numbering remains constant. This is achieved by introducing a contrast parameter to the void domain, similar to standard SIMP topology optimization and, to some extend, the Finite Cell method(Düster et al. 2008).

Within the field of level set based structural optimization, several works have been published using a similar modelling technique to that presented here. For example, in Van Miegroet and Duysinx (2007) the authors present a similar XFEM level set method, with the major difference that the void domain is omitted from the analysis. More closely related to this study is the work of Wei et al. (2010) which employs an XFEM finite element technique similar to that presented here. However, their method differs in the design field representation, which uses radial basis functions, and in the way the regularization is introduced to the design problem. More recent publications include the work of Villanueva and Maute (2017) which utilize classical XFEM with enrichments. Furthermore, the ghost penalty stabilized CutFEM method by Burman et al. (2014) has been used for level set shape optimization using an advection-diffusion design update in Burman et al. (2018) and Bernland et al. (2018), and based on an optimality criteria in Burman et al. (2019).

In this paper we present a generalized shape and topology optimization approach, in which, we utilize well-known and thoroughly tested tools from the density method to construct a crisp interface level set type design method. That is, from the density methods we employ the design field representation, image processing filters for regularization (Sigmund and Torquato 1996; Bourdin 2001; Guest et al. 2004) and the socalled robust approach (Sigmund 2009; Wang et al. 2011) to ensure a minimum feature size. The resulting optimization problem is solved by mathematical programming using the Method of Moving Asymptotes (MMA) (Svanberg 1987). The mathematical design field is introduced as a nodal field, which after a series of filters and projections is mapped to a level set field. Thus, there is a clear link between the between the nodal density representation and the level set interpretation. This approach allows us to introduce length scale control, and hence robustness, to the design problem using the well-known robust formulation from Wang et al. (2011).

We remark that other length-scale approaches originally developed for density methods already have been employed for level set optimization, e.g. the geometric constraint of Zhou et al. (2015) is used by Jansen (2018). It is also important to mention that other feature size approaches exist which are especially developed for level set methods c.f. Chen et al. (2008), Dapogny et al. (2017) and Yamada (2019), which uses an energy method, a distance penalization and a fictitious 


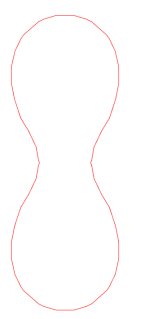

(a)

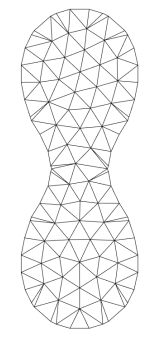

(b)

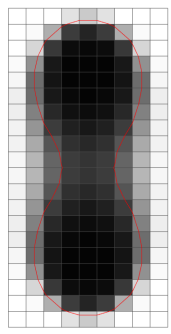

(c)

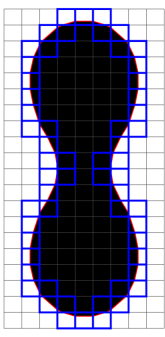

(d)

Fig. 1 Illustration of different ways to represent an hourglass type geometry in structural optimization. Plot (a) show the true geometry and (b) shows a body fitted mesh of the hourglass. In (c) the standard ersatz material / density model is shown, while (d) shows the cut element approach where the cuts are highlighted in blue.

physical model, respectively. An alternative to feature size control is to include uncertainty in loads, geometry and material parameters. This has been studied for density methods in (Schevenels et al. 2011) and for level set methods in (Chen et al. 2010; Chen and Chen 2011). We remark that this is often also termed robust design, however, in this work robust refers to designs that are insensitive to uniform over and under etching.

To summarize the contributions to the field of structural optimization, this paper presents; 1) a robust level set formulation for compliance minimization with controllable length scale, 2) a level set optimization formulation based on density method ideas and tools, 3) a level set formulation capable of solving mechanism design problems such as the compliant force inverter and 4) a simple, engineering take on the implementation of a cut element methodology.

Finally, we remark that although the motivation for this work stems from problems that require a high degree of accuracy in its interface representation, we only present numerical examples based on simpler and well known optimization problems from solid mechanics. Hence, it is not the purpose of this paper to present a faster and more efficient method than the density method for this specific class of problems. The goal is rather to demonstrate how little effort is required to go from a density method to a level set method.

The remainder of the paper is organized as follows. First we present the cut element method used to solve the linear elasticity equations. Next, the design representation and the image processing filtering schemes are introduced. This is followed by a step by step presentation of the overall optimization procedure. The methodology is then applied to several numerical examples after which the findings are summarized and discussed. To facilitate an easy step into optimization using cut elements for researchers in the community we provide the base code for download from our webpage www.topopt.dtu.dk ready for multiphysics extensions.

\section{Physical and numerical model}

Since the purpose of this work is to demonstrate and highlight the adaption of density based techniques into a level set approach, the physics is chosen as the origin of structural optimization, namely, linear elasticity. More specifically, we consider linear elasticity under an isotropic, plane stress assumption for which the governing equations and constitutive law is given as

$$
\begin{aligned}
\nabla \cdot \boldsymbol{\sigma} & =\mathbf{0} \quad \text { in } \Omega \\
\boldsymbol{\sigma} \boldsymbol{n} & =\boldsymbol{t} \quad \text { in } \Gamma_{N} \\
\boldsymbol{u} & =\boldsymbol{u}^{*} \quad \text { in } \Gamma_{D} \\
\boldsymbol{\sigma} & =\mathbf{C} \boldsymbol{\epsilon} \\
\boldsymbol{\epsilon} & =\frac{1}{2}\left(\nabla \boldsymbol{u}+\nabla \boldsymbol{u}^{T}\right)
\end{aligned}
$$

where $\boldsymbol{\sigma}, \boldsymbol{\epsilon}$, and $\boldsymbol{u}$ are the Cauchy stress, strain and displacement, respectively and $\mathbf{C}$ is the plane stress constitutive law (Cook et al. 2002). The load and boundary conditions are comprised of surface tractions, $\boldsymbol{t}$, prescribed displacements $\boldsymbol{u}^{*}$ and point loads.

The choice of numerical strategy for the governing equations are, within the context of structural optimization, most often determined by the type of optimization employed. For example, in classical shape optimization the domain is most often discretized by a body fitted mesh as seen in Figure 1(b). This provides an accurate modelling of the physics at the price of repeated and expensive re-meshing or mesh updating schemes, e.g. (Allaire et al. 2014; Christiansen et al. 2014). Though this approach is highly accurate, it can become cumbersome when performing large-scale, parallel computations. On the other hand, in classical density based topology optimization the geometry is often mapped to a structured background mesh, c.f Fig. $1(\mathrm{c})$, which in turn means that both structural and void phases must be modelled. Here, the two-phase model is handled by introducing an artificial stiffness in the 
void region such that $E_{\text {solid }} \gg E_{\text {void }}$. This results in a so-called immersed boundary method which makes the book keeping easier and eliminates the need for remeshing. However, it comes at the prize of reduced accuracy in the modelling of the interface between solid and void, either due to the presence of intermediate densities (ersatz material model) or by introducing a jagged or stair-cased interface. This can cause issues with the application of pressure loads, traction forces and other boundary dependent quantities.

In this work we adapt a crisp boundary method similar to Finite Cell method, and to some extend also the CutFEM and XFEM methods for material/void modelling. We choose the term cut element method for this modelling approach, as this name implies elements are simply cut and that no enrichments or stabilization terms are introduced. In summary, we introduce a fixed, structured background mesh using linear quadrilaterals and allow for an exact boundary representation, c.f Fig. $1(d)$, by triangulation and careful integration of the elements containing the immersed boundary.

\subsection{Discretization}

The cut element formulation is obtained by discretizing the weak form of the governing equations in (1)-(3) and at the same time introducing an indicator field $\alpha(\boldsymbol{x})$, which is independent of the underlying mesh. The indicator function is constructed such that a point which is inside $\Omega_{s}$ will yield $\alpha(\boldsymbol{x})=1$ while in $\Omega_{v}$ is assigned $\alpha(\boldsymbol{x})=10^{-9}$. This is seemingly very similar to the standard density method, however, it is important to note that the cut element method does not employ a standard ersatz material model by averaging nodal or element density variables. Instead it ensures, by the careful integration presented in section 2.2, that the solid domain is modelled exactly such that the result will coincide precisely (down to discretization error) with results obtained by modelling only the solid phase. In turn, this also means that the solution in the fictitious region can - in principle - take on any value needed to ensure the accuracy of the immersed body. This is an important property of the method and has the following effect when considering multiple material phases or multiple physics. Each material phase, or physical model, requires its own complete background mesh to allow for an accurate model description of e.g. jumps in material coefficients, which means that the total number of dofs becomes a multiple of the chosen material phases or included physics. In other words, this approach is not monolithic.
Following the proposed cut element approach, a discrete weak form of (1)-(3) is obtained as

$$
\sum_{e}^{n_{N}} \int_{\Omega^{e}} \alpha(\boldsymbol{x}) \boldsymbol{\epsilon}\left(\boldsymbol{v}_{e}\right): \boldsymbol{\sigma}\left(\boldsymbol{u}_{e}\right) \mathrm{d} \Omega-\sum_{e}^{n_{M}} \int_{\Gamma_{N}^{e}} \boldsymbol{v}_{e} \cdot \boldsymbol{t} \mathrm{d} \Gamma=0
$$

where $n_{N}$ is the number of elements in the mesh, $n_{M}$ is the number of traction boundary elements, $\boldsymbol{v}_{e}$ is the element test function and : refers to the double-dotproduct. Throughout the presented work we use 4-node quadrilateral elements with linear Lagrange shape functions for the background mesh. After the application of a standard finite element assembly operator, one arrives at the following linear system of equations

$\sum_{e}^{n_{N}} \mathbf{k}_{e}(\alpha(\boldsymbol{x})) \mathbf{u}_{e}=\sum_{e}^{n_{M}} \mathbf{f}_{e} \Rightarrow \mathbf{K}(\alpha) \mathbf{u}=\mathbf{F}$

The element matrix and traction load vector is obtained by numerical integration using standard Gaussian quadrature (Cook et al. 2002). In the following we assume, without loss of generality, that all traction forces are applied to interfaces that coincides with the boundaries of the underlying mesh. Thus, only the elements intersected by the immersed boundary needs to be treated using the cut element approach. In order to present the element integration scheme in a single, unified mathematical description capable of covering both quadrilaterals (background mesh) and triangles (cut elements), we chose to collect each pair of quadrature points in a list, instead of the double sum most often used for quadrilateral elements (Cook et al. 2002), i.e.

$\mathbf{k}_{e}=\sum_{i=1}^{n_{I P}} \alpha_{i} \mathbf{B}\left(\xi_{i}^{q}, \eta_{i}^{q}\right)^{T} \mathbf{C B}\left(\xi_{i}^{q}, \eta_{i}^{q}\right) W_{i}$

where $\left(\xi_{i}^{q}, \eta_{i}^{q}\right)$ are the $i$ 'th set of quadrature points. The remaining parts $\alpha_{i}, \mathbf{B}\left(\xi_{i}^{q}, \eta_{i}^{q}\right)$ and $W_{i}$ are the indicator function, strain-displacement matrix, and the combined weight factor at integration point $i$, respectively. $\mathbf{C}$ is the plane stress constitutive matrix, which is kept constant for all integration points. The superscript $(\cdot)^{q}$ refers to the Gauss point belonging to a quadrilateral iso-parametric element and is included to allow for a transparent formulation which later will include the cut (i.e. triangular) element integration. The integer $n_{I P}$ refers to the number of integration coordinates in the element which, for completeness, are stated here for a bilinear quadrilateral element requiring 4 sets of points: $\left(\xi_{i}^{q}, \eta_{i}^{q}\right)=( \pm 1 / \sqrt{3}, \pm 1 / \sqrt{3})$ with weights $w_{\xi_{i}}^{q}=w_{\eta_{i}}^{q}=1$. Thus, the weight factor for the quadrilateral element is given by

$W_{i}=w_{\xi_{i}}^{q} w_{\eta_{i}}^{q} \operatorname{det}\left[J^{q}\left(\xi_{i}^{q}, \eta_{i}^{q}\right)\right]$ 

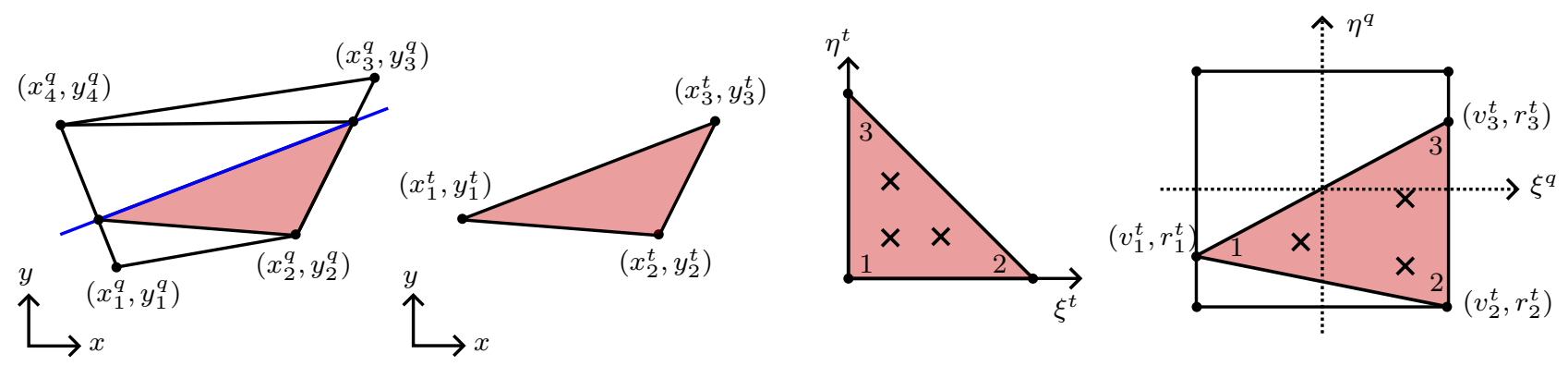

Fig. 2 Graphical representation of the cut-element integration scheme. The background element is cut by the blue line and a triangulation is performed, resulting in four sub-triangles. The integration points for the cut element are then obtained based on the quadrature points of the isoparametric triangular sub-elements, which are mapped back to the reference quadrilateral element. Each integration point is weighted by the relative area of the sub-element.

We remark, that for all elements which are completely in the solid or void regions, the presented integration scheme is exactly the same as that used in classical density based topology optimization, c.f. (Sigmund 2001a). The only difference appears at the cut elements which are treated separately in Sections 2.2 and 3.2. Finally, throughout the presented work we enforce Dirichlet boundary conditions and point loads by aligning the background mesh and the points of interest by construction. However, arbitrary conditions can be enforced using Nitsche's method (Nitsche 1971), though this is deemed outside the scope of this manuscript.

\subsection{Cut element integration}

The accuracy of the cut element approach relies on a special integration scheme applied to all cut elements in the mesh, c.f. the elements marked with a blue outline in Fig. 1(d). First all the cut elements are identified and then triangulated individually as shown for a single element in Fig. 2(left) where the blue line refers to the cut. Once the element triangulation is obtained, the cut element scheme consists of mapping the three triangular Gauss points back into the reference quadrilateral element as shown in the Fig. 2(right), computing a modified weight relative to the area of the sub-triangle and performing the integration scheme from eq. (6) with the appropriate $\alpha_{i}$. This process is repeated for each subtriangle of the cut element and their contributions are summed to yield the element stiffness matrix. Thus, for an element with a single cut, four triangles appear resulting in a total of 12 integration points. For the rare case of double-cut elements six triangles would appear resulting in 18 integration points. Finally we emphasize that the size of the resulting element stiffness matrix remains 8 by 8 for the four node reference quadrilateral.

To summarize, the only thing needed to perform the cut element integration is the mapping from the sub-triangle to the reference quadrilateral. This allows for all operations to be performed in the iso-parametric space assuming that the triangulation is available in local coordinates, c.f. section 3.2. The coordinates spanning each sub-triangle are denoted by $\left(v_{i}^{t}, r_{i}^{t}\right)$, where superscript $t$ refers to the triangle and $i \in\{1,2,3\}$. The position of the quadrature points in the local quadrilateral, can now be obtained using the shape function for a linear triangle, i.e.

$\xi_{i}^{q}=v_{1}^{t}\left(1-\xi_{i}^{t}-\eta_{i}^{t}\right)+v_{2}^{t} \xi_{i}^{t}+v_{3}^{t} \eta_{i}^{t}$
$\eta_{i}^{q}=r_{1}^{t}\left(1-\xi_{i}^{t}-\eta_{i}^{t}\right)+r_{2}^{t} \xi_{i}^{t}+r_{3}^{t} \eta_{i}^{t}$

The local quadrature points for a linear triangle are given by $\xi^{t}=\left\{\frac{1}{6}, \frac{2}{3}, \frac{1}{6}\right\}$ and $\eta^{t}=\left\{\frac{1}{6}, \frac{1}{6}, \frac{2}{3}\right\}$. Finally, the weight used for each integration point in the quadrilateral must be modified to include the dependence of the triangular weights $w^{t}=\left\{\frac{1}{3}, \frac{1}{3}, \frac{1}{3}\right\}$ as well as the area ratio between the triangle and the reference element i.e.

$W_{i}=w_{i}^{t} \operatorname{det}\left[J^{t}\left(\xi_{i}^{t}, \eta_{i}^{t}\right)\right]=w_{i}^{t} A_{q}^{t} \operatorname{det}\left[J^{q}\left(\xi_{i}^{q}, \eta_{i}^{q}\right)\right]$

where $\operatorname{det}\left[J^{t}\left(\xi_{i}^{t}, \eta_{i}^{t}\right)\right]$ refers to the determinant of the Jacobian for the triangular element and $A_{q}^{t}$ is the area of the considered triangle in the local quadrilateral coordinate system. This means that the sum of all $A_{q}^{t}$ 's in a cut element is 4 due to the construction of the isoparametric quadrilateral element. To ease implementation we employ the right hand side of eq. (10) in the presented work, since this alleviates the need to compute the coordinates of the sub triangles, i.e. points $\left(x_{i}^{t}, y_{i}^{t}\right)$ in Fig. 2 .

It should be noted that pure solid and void element matrices can be pre-computed as in density based methods. Only the cut elements must be integrated at every optimization iteration both for analysis and sensitivity computation. This leaves a slightly higher computational cost per optimization iteration, which may be alleviated by compiling the element integration routines using e.g. MEX in Matlab. In general we have observed 


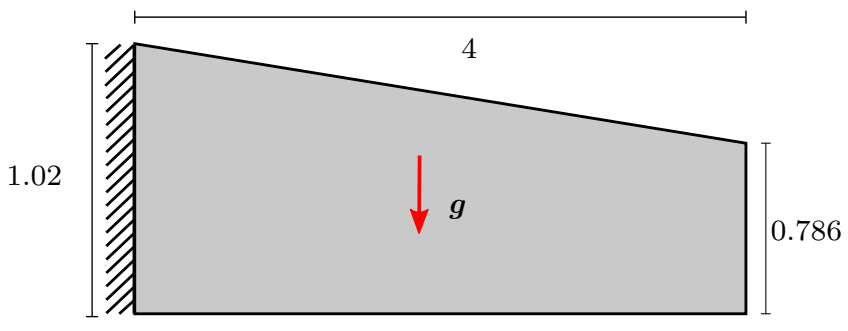

Fig. 3 Cantilever model problem used to demonstrate the cut element approach. The dimensions are as stated in the figure and the cantilever is subjected to a gravitational load with $\boldsymbol{g}=\{0,-1\}$. The problem is solved on a 4 by 3 domain with a background mesh of $43 \times 32$ elements for various positions of the cantilever.

that the extra cost is by no means excessive, and that most time is still spend on solving the equation system.

\subsection{Model verification}

To demonstrate the validity of the cut element method for arbitrary element intersections we use the model cantilever problem with dimension as sketched in Fig. 3 loaded by a gravitational force of $\boldsymbol{g}=\{0,-1\}$. The idea is to let the inclined cantilever move through a background mesh of size 4 by 3 , discretized by $43 \times 32$ elements. The odd numbers are chosen to minimize the risk of repeating the same cut situations for every analysis. The cantilever is then moved from $\mathrm{y}=0.3438$ to $y=1.7575$ in 63 step and for each analysis the compliance is computed. Fig. 4 shows the displacement magnitude at two instances, and the relative error is shown in Fig. 5. The maximum relative error in compliance is found to be $1.17 \times 10^{-4}$ and we note that this number goes down with mesh refinement. Although the presented work solely uses direct methods for the linear solves, we also investigated the condition number of the system matrices and found that these vary between $1.5 \times 10^{12}$ and $3.5 \times 10^{12}$. We remark, that the condition numbers did not follow the same pattern as the compliance variations in Fig. 5. When compared to the condition numbers encountered in density based immersed boundary methods (using top $88 . \mathrm{m}$ ), we find that these are of the same order. This means that the efficient parallel preconditioner presented in e.g. Amir et al. (2014) also works excellent for the presented cut element method.

\section{Design parameterization}

The design parametrization is introduced to the problem as a scalar nodal design field $s \in[0 ; 1]$, exactly

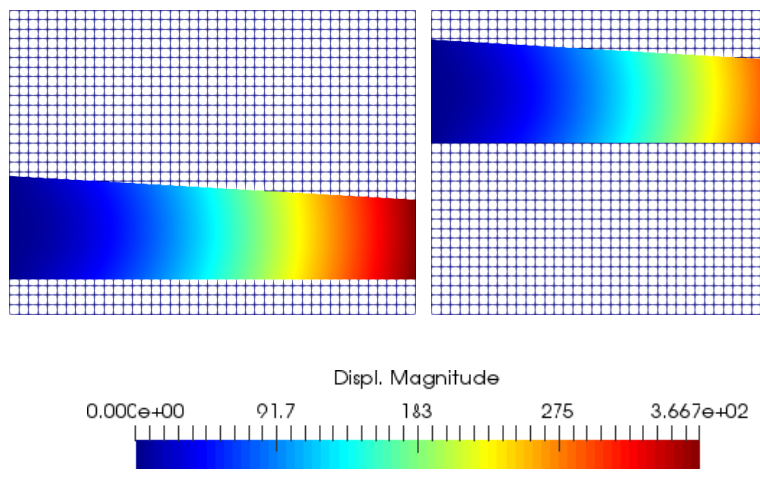

Fig. 4 Displacement field for two positions of the cantilever from Fig. 3 in a large, fixed background mesh.

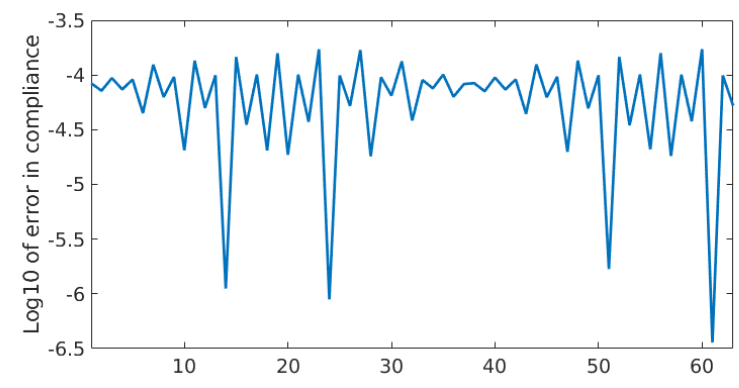

Fig. 5 Error $\log _{10}(\varepsilon)$ of the relative error in the compliance for 63 different cantilever positions along the y-axis, c.f. Fig. 3

as done in nodal density methods, e.g. (Guest et al. 2004). A generic example is shown in the top left of Fig. 6, which also depicts the mapping and filter operations that lead to the physical level set field used for the finite element analysis. The interface between solid and void is from here on defined as the zero contour of the level set field, such that $\phi_{i}>0$ refers to solid and $\phi_{i}<0$ to void. In the following sections we introduce the filter operations, cut element triangulation and design sensitivity calculation, respectively.

\subsection{Projection filter}

If a linear mapping is used to map the design variables to the level set values, the support for the level set is very compact and the level set function may oscillate and small, localized features would appear. This can partly be alleviated by the introduction of a regularization scheme, i.e. the smoothing filters known from density based topology optimization (Bourdin 2001). This increases the support of the level set field and results in a smoother boundary representation as well as a more stable optimization process. The convolution type filter 


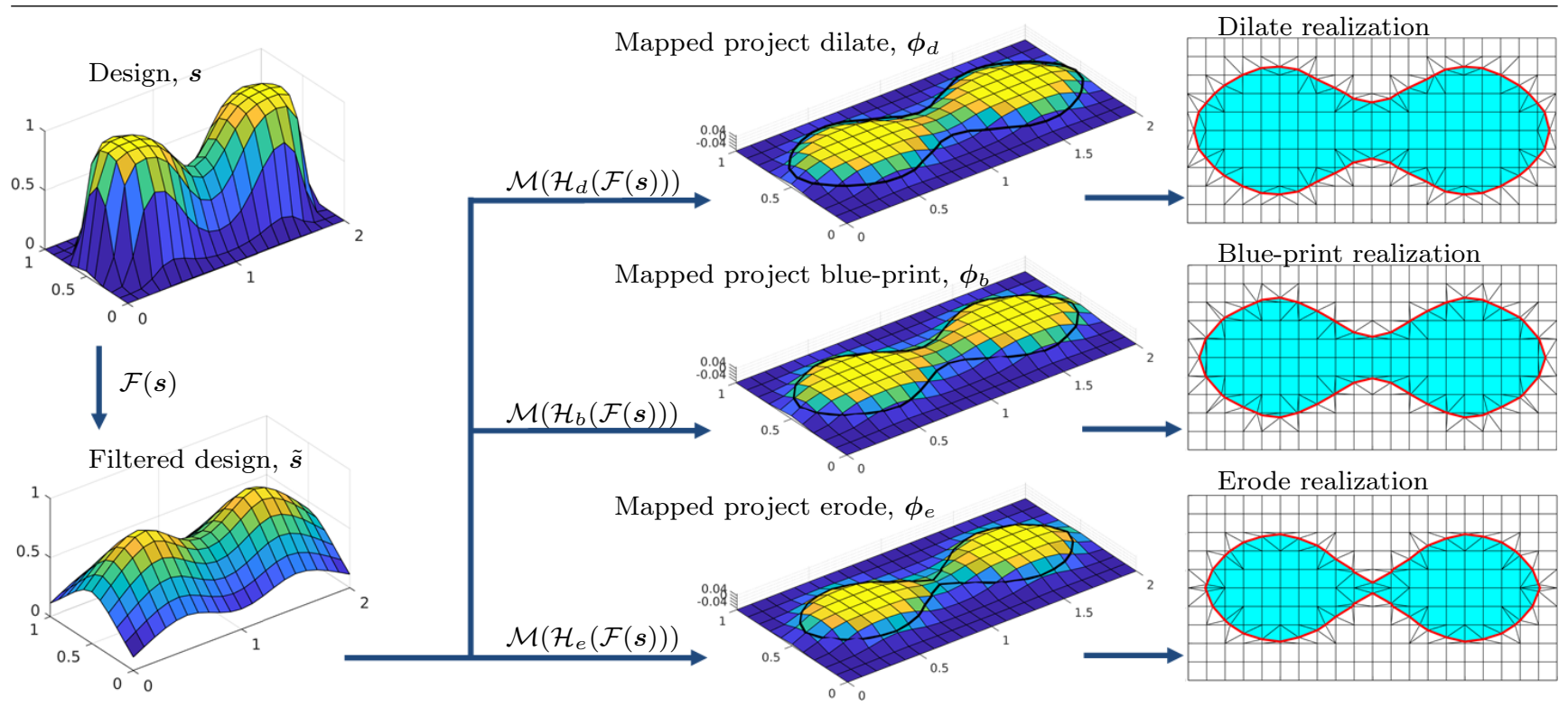

Fig. 6 Overview of the design parametrization. Nodal design variable field $s$, filtered field $\tilde{s}$, design projected and mapped to level set for three realizations and finally the mesh with visualization of the sub-triangles in the cut-elements.

can be expressed as

$\tilde{s}_{i}=\frac{\sum_{j \in N_{i, j}} s_{j} w\left(\boldsymbol{x}_{j}\right)}{\sum_{j \in N_{i, j}} w\left(\boldsymbol{x}_{j}\right)} \Rightarrow \tilde{\boldsymbol{s}}=\mathcal{F}(\boldsymbol{s})$

where $N_{i, j}$ is the neighborhood set of nodes within the filter radius $R$ and $w\left(\boldsymbol{x}_{j}\right)$ is the linear weight function between two nodal points $\boldsymbol{x}_{j}$ and $\boldsymbol{x}_{i}$, i.e.

$w\left(\boldsymbol{x}_{j}\right)=R-\left|\boldsymbol{x}_{j}-\boldsymbol{x}_{i}\right|$

To introduce the possibility of operating with multiple physical realizations using a single design field, and to suppress small localized features, a projection filter is introduced. This filter is identical to the - by now - classical robust formulation from density methods (Wang et al. 2011; Lazarov et al. 2016). Considering a smoothed design, the projection of this design can be obtained using a smoothed approximation of the Heaviside function

$\hat{\boldsymbol{s}}=\mathcal{H}(\mathcal{F}(\boldsymbol{s}))=\frac{\tanh (\beta \eta)+\tanh (\beta(\tilde{\boldsymbol{s}}-\eta))}{\tanh (\beta \eta)+\tanh (\beta(1-\eta))}$

where $\eta$ is the threshold value and $\beta$ is the steepness of the projection. For multiple projections of the same design we may refer to the eroded, blue-print and dilated realizations using subscripts $e, b, d$ and corresponding threshold values $\eta_{d} \leq \eta_{b} \leq \eta_{e}$, respectively. For the numerical examples we use a variable off-set $\Delta \eta$ which yields the erode and dilate threshold values as $\eta_{e}=$ $\eta_{b}+\Delta \eta$ and $\eta_{d}=\eta_{b}-\Delta \eta$, respectively.

After the filter and projection operators, the physical level set is obtained by a linear mapping of the design field, which ensures that the level set is bounded by $\phi \in\left[\phi_{\min } ; \phi_{\max }\right]$ yielding

$\phi=\mathcal{M}(\mathcal{H}(\mathcal{F}(s)))=\phi_{\min }+\left(\phi_{\max }-\phi_{\min }\right) \hat{\boldsymbol{s}}$

The interval of the physical level set is chosen as $\phi \in$ $[-h / 2 ; h / 2]$, where $h$ is the side length of an element, following the work of (Sharma and Maute 2017). Also, this scaling has the beneficial effect of rendering the design update mesh independent.

\subsection{Triangulation of cut elements}

The only step missing to complete the proposed level set based modelling is the triangulation of the cut elements. In this work the cut elements are triangulated directly based on the nodal design information using the marching squares algorithm, which is a straight forward simplification of the marching cubes algorithm by Lorensen and Cline (1987). That is, based on the nodal values of the level set field, we employ the marching squares algorithm to obtain the positions of the cut element edges in local quadrilateral coordinates. This corresponds to the two points $\left(v_{1}^{t}, r_{1}^{t}\right)$ and $\left(v_{3}^{t}, r_{3}^{t}\right)$ shown in Fig. 2(right). Having obtained the positions of the cuts, the subsequent triangulation is performed without calling an external mesher. This is viable due to the simplicity of the marching squares algorithm that only contains 16 unique scenarios for the cuts. An illustrative example of the cut process is shown in Fig. 7 where only the top left node has a positive level set value whereas the remaining three are negative. The algorithm computes 
the triangulation and returns intersection points with the parent quadrilateral edges, connectivity and phase information for the triangulation. This means that the resulting representation of the boundary is piece-wise linear and therefore that the resolution of the background mesh has a significant influence on the ability to represent fine details.

Finally, we note that the sub-triangulation could have been obtained using e.g. Delauney triangulation. However, for simple 2D problems, as those investigated in this work, this did not improve the quality of the numerical solution. For 3D applications this may be a more involved task where dedicated software for meshing would be necessary.

\section{Optimization formulation}

The optimization problems investigated in this work can all be cast as a standard mathematical program, i.e.

$$
\begin{aligned}
\min _{\boldsymbol{s} \in \mathbb{R}^{n}}: & \Psi(\boldsymbol{s}, \mathbf{u}) \\
\text { subject to: } & \mathbf{K}(\boldsymbol{s}) \mathbf{u}=\mathbf{f} \\
& g_{j}(\boldsymbol{s}, \mathbf{u}) \leq 0, \quad \text { for } \quad j=1 . . m \\
& 0 \leq s_{i} \leq 1, \quad \forall i
\end{aligned}
$$

in which $\Psi(\boldsymbol{s}, \mathbf{u})$ is the objective function to be minimized over $s, g_{j}(s, \mathbf{u})$ are the $m$ potential inequality constraints and the last line corresponds to a box constraint on the design variables. The optimization problem in eq. (15) is solved by a nested approach, meaning that the state equations are considered implicit and only evaluated through the objective or the state dependent inequality constraints. The problem is solved iteratively by the Method of Moving Asymptotes (MMA) (Svanberg 1987) and details of the used internal MMA parameters are given in section 7 . The use of gradient based optimization methods require the computation of design sensitivities which are presented next.

\subsection{Evaluation of design sensitivities}

In this work we follow, as much as possible, the standard discrete adjoint sensitivity analysis used for most density based methods. In fact, the only noticeable difference is the slightly more involved evaluation of the system matrix differentiated wrt. the design variable, as will be made clear in the following.

Before proceeding, we remark that variational shape derivatives, an often used sensitivity analysis tool for level set methods, also could have been used (Choi and
Kim 2005). However, in combination with a parametric level set description, the sensitivities localized on the interface would afterwards need to be extrapolated (velocity field extension) to the nodal design variables.

For completeness, and to illustrate the similarity to density method sensitivity analysis, we provide a detailed description in the following. First, the adjoint system is obtained by forming a Lagrangian and differentiating wrt. the $i$ 'th level set variable, i.e.

$$
\begin{aligned}
L & =\Psi+\boldsymbol{\lambda}^{T}(\mathbf{K u}-\mathbf{f}) \\
\frac{\mathrm{d} L}{\mathrm{~d} \phi_{i}} & =\frac{\partial \Psi}{\partial \phi_{i}}+\boldsymbol{\lambda}^{T} \frac{\partial \mathbf{K}}{\partial \phi_{i}} \mathbf{u}+\left(\frac{\partial \Psi}{\partial \mathbf{u}}+\boldsymbol{\lambda}^{T} \mathbf{K}\right) \frac{\mathrm{d} \mathbf{u}}{\mathrm{d} \phi_{i}}
\end{aligned}
$$

By requiring the last term to be zero the adjoint problem is formulated and is noted to be identical to that from density methods.

$\mathbf{K}^{T} \boldsymbol{\lambda}=-\left(\frac{\partial \Psi}{\partial \mathbf{u}}\right)^{T}$

Now the sensitivity with respect to the level set variable $\phi_{i}$ is given by

$$
\frac{\mathrm{d} L}{\mathrm{~d} \phi_{i}}=\frac{\partial \Psi}{\partial \phi_{i}}+\boldsymbol{\lambda}^{T} \frac{\partial \mathbf{K}}{\partial \phi_{i}} \mathbf{u}=\frac{\partial \Psi}{\partial \phi_{i}}+\boldsymbol{\lambda}^{T} \sum_{e}^{n}\left(\frac{\partial \mathbf{k}_{e}}{\partial \phi_{i}}\right) \mathbf{u}
$$

where the effect of using nodal variables is observed as a need to sum over neighboring elements for each nodal point. The last ingredient in the sensitivity computation is, however, not as simple as for the density method, i.e. the calculation of the element stiffness sensitivity $\partial \mathbf{k}^{e} / \partial \phi_{i}$. First of all, for all uncut elements the differentiated element matrix is zero. In other words, the derivatives are localized around the interface between solid and void. This means that only cut elements need to be considered in the calculation. The element matrix derivative of each cut finite element is then found by a perturbation of the level set values as this involves at most 4 extra element integrations depending on the type of cut. We remark that rigorous analytic differentiation of a cut element matrix would require the computation and assembly of an element matrix for the derivative of each integration point, which by far exceeds the computational cost for perturbation (see (Sharma et al. 2017) for an in-depth comparison of various shape sensitivity approaches).

To illustrate this property of the element matrix derivative, a generic situation for a single cut element is shown in Fig. 7, including its sub-triangulation and virtual cut nodes. For this specific case it is only necessary to compute perturbations of $\phi_{1}, \phi_{3}, \phi_{4}$ as the sensitivity wrt. $\phi_{2}$ is zero since the element edges connected to node 2 are uncut. Also, in order to ensure that the triangulation of the individual element does 


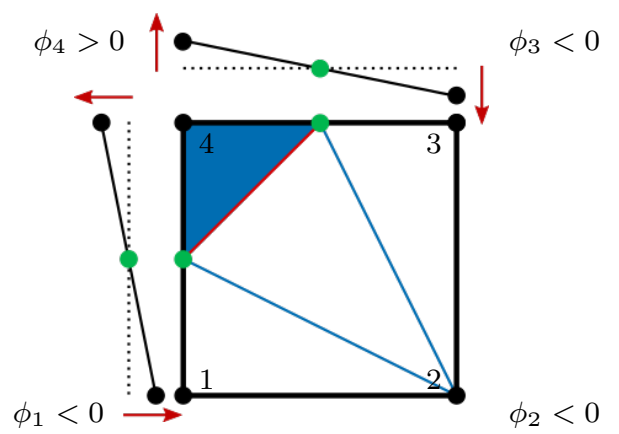

Fig. 7 Illustration of the finite difference method applied to identify the derivative of the element matrix w.r.t. the level set field. Red line is the cut defined by the two green virtual nodes. Red arrows indicate the direction of the perturbation applied.

not change by the perturbation of the level set, the nodal values are always perturbed further away from the zero level set as indicated by the red arrows in the figure i.e. $\phi_{i}^{*}=\phi_{i}(1+\delta)$ where $\delta$ is a small number, here $\delta=10^{-6}$. Based on numerical experience this value of $\delta$ has demonstrated to be a proper choice with respect to accuracy of the finite difference approximation. The element stiffness matrix sensitivity can then be computed as

$\frac{\partial \mathbf{k}_{e}}{\partial \phi_{i}}=\frac{\mathbf{k}_{e}-\mathbf{k}_{e *}}{\delta \phi_{i}}$

where $\mathbf{k}_{e *}$ denotes the perturbed element stiffness matrix. In the above derivation the sensitivities were computed wrt. the nodal values of the level set field and not the actual mathematical design variables $s$. To obtain the needed sensitivity, the chain-rule is applied to take into account the filter operations and the process is exactly the same as for density methods.

$\frac{\partial \Psi}{\partial s_{j}}=\sum_{i \in N_{j, i}} \frac{\partial \Psi}{\partial \phi_{i}} \frac{\partial \phi_{i}}{\partial \hat{s}_{i}} \frac{\partial \hat{s}_{i}}{\partial \tilde{s}_{i}} \frac{\partial \tilde{s}_{i}}{\partial s_{j}}$

or in terms of mapping operators

$$
\frac{\partial \Psi}{\partial s}=\mathcal{F}^{T}\left(\mathcal{H}^{T}\left(\mathcal{M}^{T}\left(\frac{\partial \Psi}{\partial \phi}\right)\right)\right)
$$

which basically consists of moving in the reverse order through the derivative of the operators in eq. (14). Finally, it is noted that the smoothing filter ensure that the otherwise localized sensitivity information is transferred to the neighboring region which is observed to improve the speed and stability of the optimization process.

\subsection{Initial design and target volume}

Constructing suitable initial configurations for level set type optimization problems that meets the target volume constraint is a challenge, especially for low volume fractions. That is, if the initial design is far greater than the target volume one has to be carefull in scaling objective and constraint values. In this work we scale the initial objective value to be 10 and use a MMA $c$ parameter of 100 for penalizing the volume constraint in the MMA sub-problem. We note that several alternative methods to deal with this issue exist, among them the adaptive scheme in which the target volume is lowered on the fly as done in e.g. Christiansen et al. (2014).

\subsection{Stopping criteria}

Choosing a stopping criteria for the proposed level set type optimization formulation is not as obvious as for standard density methods, in which a maximum change of $1 \%$ in the mathematical design field, $s$, is often used. For the proposed method, even a very small change in the mathematical design field can lead to a large change in the physical design field $\hat{\boldsymbol{s}}$. Our numerical experiments have shown that a stopping criteria based on the maximum change in the physical field is an appropriate choice. In the following we choose $\left\|\hat{\boldsymbol{s}}_{k}-\hat{\boldsymbol{s}}_{k-1}\right\|_{\infty}<0.01$ as a condition to stop the optimization process.

\section{Numerical examples}

In this section the proposed density based level set methodology is demonstrated on four design problems. Three minimum compliance problems, i.e. the cantilever, the MBB beam and a (1D) bar problem as well as a compliant mechanism problem which is intrinsically difficult for crisp interface level set (or pure shape) design representations. For the minimum compliance problems we also introduce a heuristic hole nucleation strategy similar to that of Christiansen et al. (2014), which is presented in section 5.1.1.

To ensure easy reproducibility of the presented method and results, we have made the Matlab code needed to run the minimum compliance problems publicly available. For the numerical examples we use the following parameters unless otherwise stated: an outer movelimit of $2 \%$, an MMA asymptote initialization of 0.5 , an MMA asymptote increase of 1.2, an MMA asymptote decrease of 0.7 , an MMA constraint penalization parameter of $c=100$, a projection parameter of $\beta=12$ and an initial objective scaling to 10 . The movelimit of $2 \%$ may seem very restrictive if seen in relation to 
density based problem settings, but as already stated, even a small change in the design variables can lead to large changes in the physical design.

When presenting the results we remark that all objective values are evaluated for the blue-print design corresponding to $\eta_{b}=0.5$, which is also the designs shown in the figures unless specified explicitly.

\subsection{Compliance minimization}

The minimum compliance problems are solved using the so-called modified robust approach for density methods presented in Wang et al. (2011). The idea is to operate with several design realizations, but at the same time utilize the nature of the compliance problem to avoid performing the FEA on each of them. The design problem can be formulated as

$$
\begin{aligned}
\min _{\boldsymbol{s} \in \mathbb{R}^{n}}: \Psi & =\int_{\Omega} \alpha^{e}(\boldsymbol{s}) \boldsymbol{\epsilon}\left(\boldsymbol{u}^{e}\right): \boldsymbol{\sigma}\left(\boldsymbol{u}^{e}\right) \mathrm{d} \Omega \\
& =\left[\boldsymbol{u}^{e}\right]^{T} \mathbf{K}^{e} \boldsymbol{u}^{e}
\end{aligned}
$$

subject to: $\mathbf{K}^{e}(\boldsymbol{s}) \mathbf{u}^{e}=\mathbf{f}$

$$
\begin{aligned}
& \int_{\Omega} \alpha^{d}(s) \mathrm{d} \Omega / V^{*}-1 \leq 0 \\
& 0 \leq s_{i} \leq 1, \quad \forall i
\end{aligned}
$$

where superscript $e$ refers to an eroded realization on which the FEA is performed and $d$ to a dilated realization for which the volume constraint is imposed. To ensure the blue-print design uses the prescribed amount of material, we perform a volume continuation scheme as presented in (Wang et al. 2011). That is, the target volume fraction for the dilated realization is updated every 20th iteration by:

$V^{*}=\frac{V^{\text {target }}}{V_{b}} V_{d}$

where $V_{b}$ and $V_{d}$ refers to the volume of the blue print and dilated designs, respectively, and $V^{\text {target }}$ is the desired target volume for the blueprint design. Finally, we note that the minimum compliance problem is selfadjoint, i.e. $\boldsymbol{\lambda}=-2 \boldsymbol{u}$, and that the sensitivity can be expressed as

$$
\frac{\mathrm{d} \Psi}{\mathrm{d} \phi_{i}}=-\sum_{e}^{n}\left(\boldsymbol{u}_{e}^{T} \frac{\partial \mathbf{k}_{e}}{\partial \phi_{i}} \mathbf{u}_{e}\right)
$$

This means that the objective sensitivity does not require the solution of an adjoint problem.

\subsubsection{Hole nucleation strategy}

By construction, the level set based parametrization cannot create new holes from inside the solid region. Therefore, a hole nucleation scheme is required if new holes should be allowed to emerge during the design evolution. An often used workaround is to start with an initial design that already contains several holes. These may potentially move, change shape or disappear during the optimization process. This, however, introduces a strong initial design dependence that for some examples can prohibit the optimizer from reaching a good quality local minimum. In order to alleviate this shortcoming, a hole nucleation scheme based on the strain energy density in the elastic body is introduced. For shape and level set based structural optimization problems, hole nucleation strategies have previously been introduced using topological derivatives, c.f. (Eschenauer et al. 1994; Sokolowski and Zochowski 1999; Feijóo et al. 2003). For the specific case of linear elasticity and minimum compliance, it is known that the strain energy density and the topological derivative are equivalent up to a constant for Poisson's ratio $\nu=\frac{1}{3}$. In this work we therefore adopt an approach close to the one presented in (Eschenauer et al. 1994; Christiansen et al. 2014), which can be expressed as follows. The level-set is initiated using a constant value slightly above zero contour such that the entire domain is solid. In order to sort the strain energy densities computed for every element $i$ in the eroded design we use $\varphi_{i}=\left[\boldsymbol{u}_{i}^{e}\right]^{T} \mathbf{k}_{i}^{e} \boldsymbol{u}_{i}^{e}$ where $\mathbf{k}_{i}^{e}$ is computed as in eq. (6). We then compute a relative energy measure

$\tau_{i}=\frac{\varphi_{i}-\varphi_{\min }}{\varphi_{\max }-\varphi_{\min }}$

At the first and every subsequent 20th iteration do the following:

1. Compute the element-wise strain energy density field.

2. Truncate the field at all void elements by assigning the max value of the field.

3. Map to nodal representation using bi-linear shape functions.

4. Compute $\boldsymbol{\tau}$ and allow hole-nucleation at nodes fulfilling $\tau_{i}<5 \cdot 10^{-4}$, however, at most 10 new holes.

5. Generate the hole(s) by perturbing the design field $s$ in the selected nodes until the dilated level set shows a hole at these specific nodes.

This process is repeated until the target volume is reached, or the current volume is below $30 \%$, after which the hole nucleation scheme is stopped. Hereafter only the target volume fraction $V^{*}$ is updated every 20th iteration using eq. (24). If not stopped, the low volume fraction designs can easily degenerate by disconnecting the load 


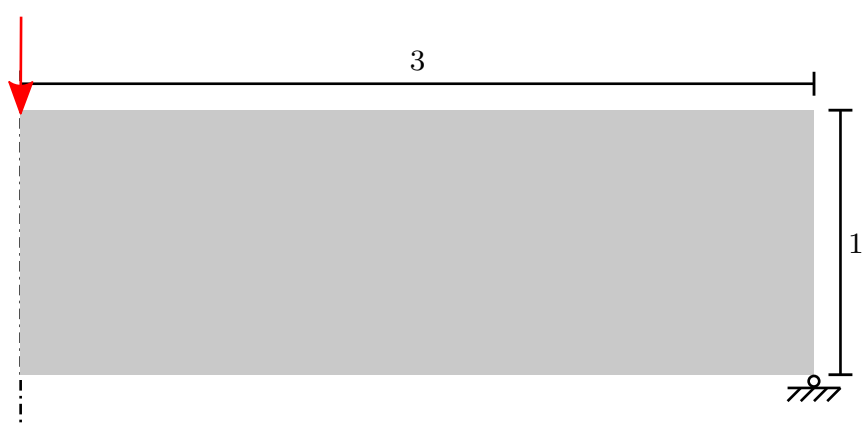

Fig. 8 The half MBB beam design problem using symmetry.

from the support. That is, since the hole nucleation size in this work is related to the filter, a single hole may cut an entire bar. We remark that the restriction to 10 simultaneous hole nucleations is based on the authors' experience through a number of numerical experiments. Inserting too many holes may disconnect the structure which, due to the nature of the hole nucleation scheme, is irreversible and may lead to optimization failure. On the other hand, inserting only a single hole may destroy symmetry of the design.

\subsubsection{The $M B B$ beam}

The first compliance example concerns the classical MBB beam design problem as depicted in Fig. 8. The design is optimized using the proposed robust formulation and hole nucleation scheme under a volume constraint of $40 \%$ solid material using the fully solid initial design in Fig. 11(a). We remark that using a fully solid initial guess, means that no shape sensitivity information is available at the first iteration. Thus, the first step of the optimization process is to introduce holes in order to create a solid/void interface from which the shape optimization can proceed. We remark that this could have been postponed by padding the design domain as suggested in e.g. (Clausen and Andreassen 2017). The material parameters are set to $E=1$ and $\nu=0.3$, the filter radius to $R=5$ nodes and the projection offset is chosen as $\Delta \eta=0.01$. Note that no continuation scheme is used for the smooth Heaviside projection filter. The resulting optimized design for a mesh resolution of 150x50 elements is shown in Fig. 9 which also shows the positions of the nucleated holes during the optimization process. In addition a plot of the optimization history is shown. From the history it is evident that the initial compliance is low, due to stiff initial design. Subsequently, the objective function increases as the design is morphing and the volume is reduced. At every 20 th iteration new holes can potentially be inserted and the volume is continuously decreasing. After 106 it-

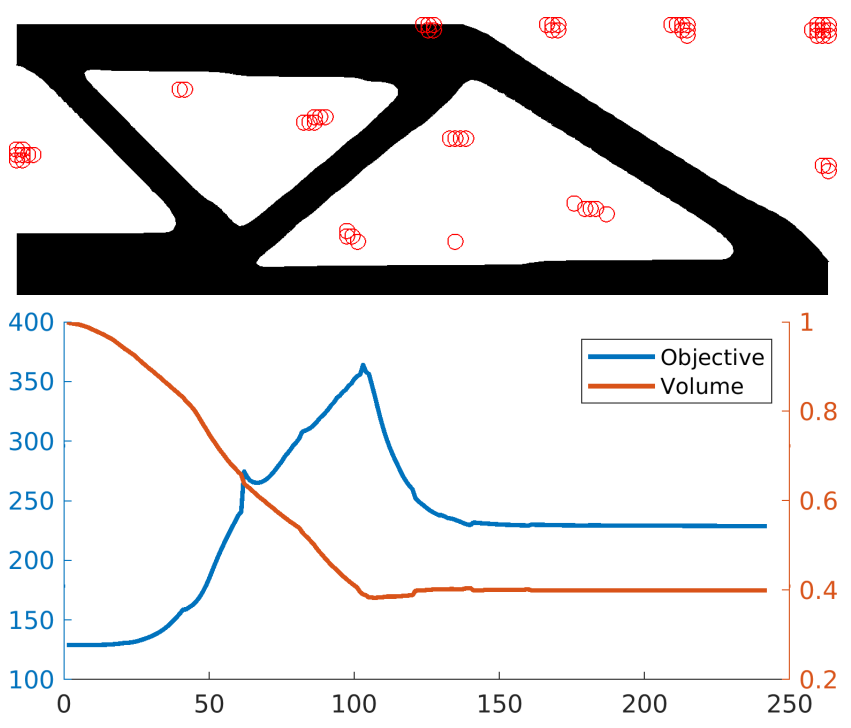

Fig. 9 MBB beam design history using the proposed hole nucleation scheme. Red circles in design plot show where holes have been nucleated during the optimization process. Below the objective function and volume-fraction history is shown. Material volume $40 \%$, filter radius $R=5$, threshold offset $\Delta \eta=0.01$. Final blue-print objective $\Psi=227.4$ after 242 iterations

erations the blue-print volume is reached and the hole nucleation is disabled. Finally, the optimization process is terminated by the stopping criterion after 242 iterations. For completeness of the robust design description, Fig. 10 shows the mathematical design field, the filtered design and the projected design, respectively, corresponding to the optimized design in Fig. 9. From these plots it is clear that the projection filter has the exact same effect in relating the mathematical design field to its physical interpretation as is observed for density methods, c.f Wang et al. (2011). That is, the physical design field (bottom) is fully converged to the upper and lower bound, i.e. 0-1, with only a small spatial gradient on the interface allowing a smooth interface description for the cut element analysis. From the center plot it is seen that the filtered field has a constant spatial gradient along the material-void interface, which is important for the length scale control i.e through $\Delta \eta$. Regarding the intermediate values of the mathematical design field (top) seen away from the interface, we remark that they play no role in the physical realization due to the cut element analysis.

If an initial design containing predefined holes is specified the optimization procedure can be used without the hole generation scheme, i.e. pure shape optimization, but with the possibility to close holes. This is investigated using the three initial designs shown in Fig. 11(b-d). While the initial configurations in Fig. 11(b) and (c) simply consist of layouts with predefined 


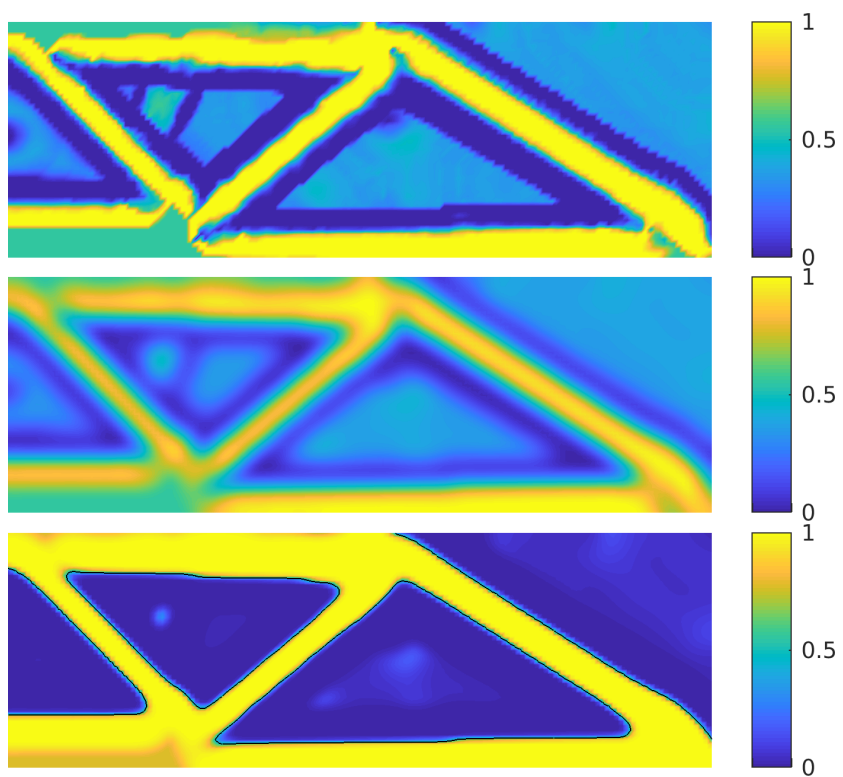

Fig. 10 Top: Design field $s$, middle: Filtered design field $\tilde{s}=\mathcal{F}(s)$, bottom: Projected design field $\hat{s}_{b}=\mathcal{H}_{b}(\tilde{s})$ for the optimized design in Fig. 9.

(a)

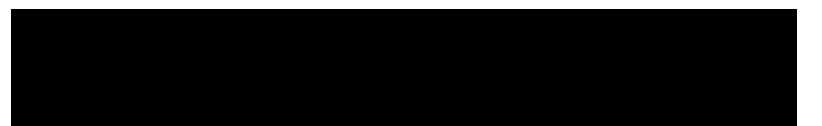

(b)

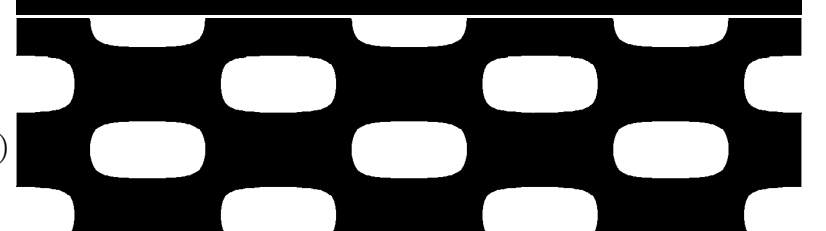

(c)

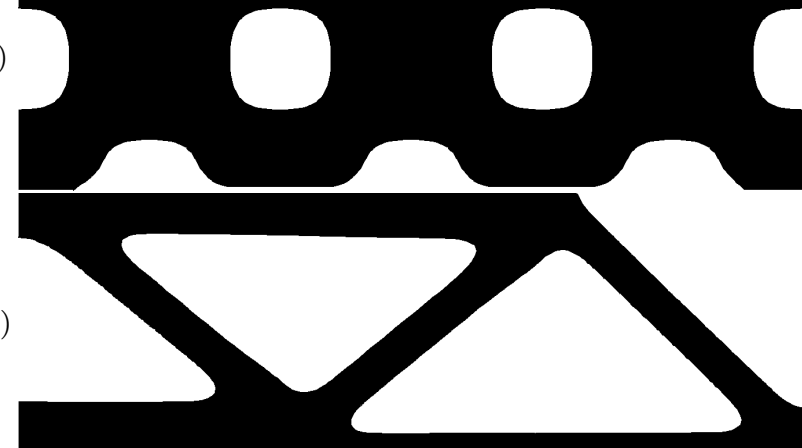

Fig. 11 Initial designs used for the optimization of the MBB beam. (a) an almost solid design, (b) a 6 by 4 hole configuration, (c) a 3 holes configuration and (d) thresholded density result using the top88 code.

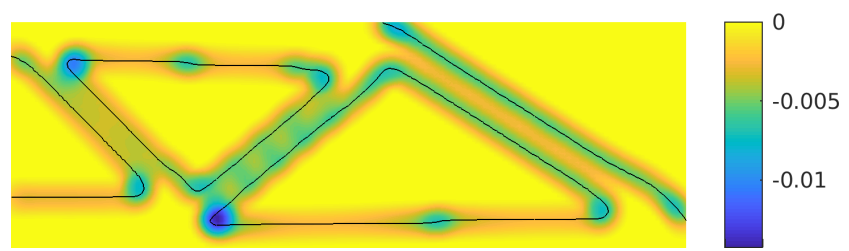

Fig. 12 Plot of the objective function sensitivities for the final design of the MBB beam in Fig 9. Sensitivities are localized near the interface.

holes, the initial design in Fig. 11(d) corresponds to an optimized design obtained using the top88 code (Andreassen et al. 2011), for which the result is mapped onto a nodal basis. The resulting optimized designs are shown in Fig. 13 and are obtained using the same parameters as those used for the design in Fig. 9. Despite similar objective function values, it is seen that the topology differs for the design obtained using the initial guess in Fig. 11(b). This demonstrates that the proposed method is, similar to other shape optimization methods, highly influenced by the initial configuration.

We note that none of the start guesses, except that obtained using top88, fulfill the volume constraint initially. A general observation is that during the process of reducing the volume, existing holes may grow and bars may disappear. However, when the target volume is reached the optimizer is more reluctant to remove bars and thus the topology tends to be more or less fixed. For the design obtained using the top88 code, the topology remains the same while the bars are slightly adjusted. It is also seen that the symmetry condition artifacts imposed by the top88 density filter, i.e. that the design is always perpendicular the boundaries, are alleviated.

\subsubsection{Ensuring a length scale}

Ensuring a length scale in an optimized design is paramount for manufacturing considerations and hence, the versatility and applicability of a given design methodology to real world engineering problems. However, for the proposed crisp interface cut element design approach there is an additional argument for its necessity. As with all other numerical design methods, the mathematical program will find and utilize all weaknesses of a given scheme. For the cut element approach the main issue has to do with sub-element features, so-called doublecuts, which results in an artificially high stiffness. In addition, the formation of narrow notches where two cut elements neighbor each other is also theoretically, as well as design-wise, dubious. To illustrate these problems the MBB problem is solved without the robust formulation and the resulting design is shown in Fig. 


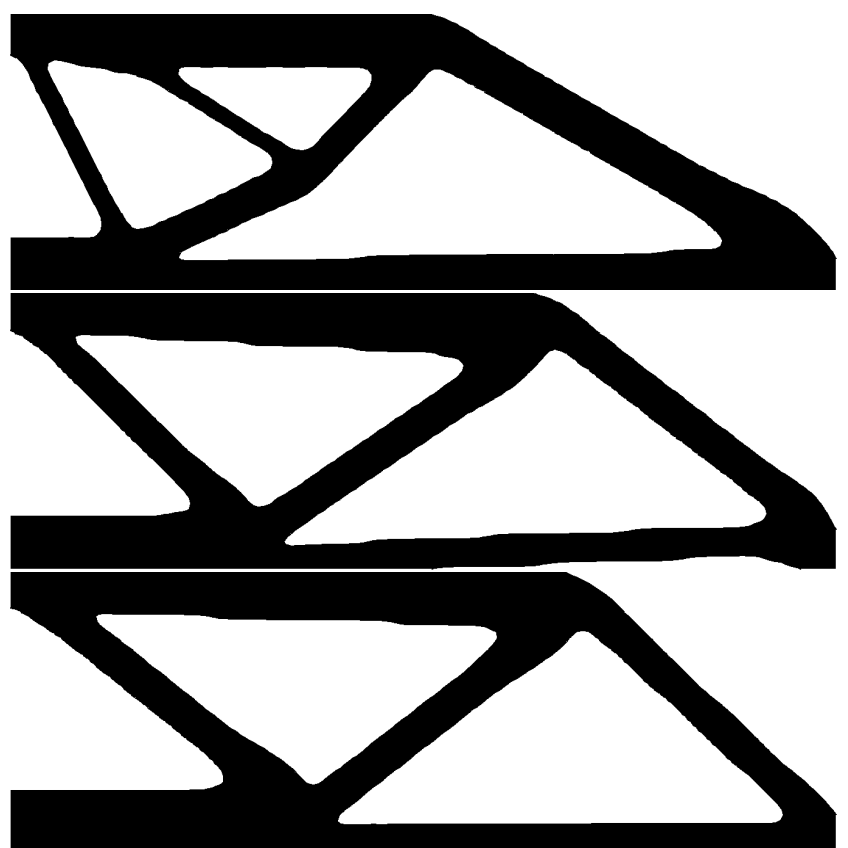

Fig. 13 Initial design dependence for the optimization of the MBB beam. $40 \%$ volume fraction Mesh $150 \times 50$ elements, $R=5$ elements, $\beta=12, \Delta \eta=0.01$. The obtained objectives are $\Psi=\{229.7,225.4,223.9\}$ using the initial designs in Fig. $11(\mathrm{~b}-\mathrm{d})$.

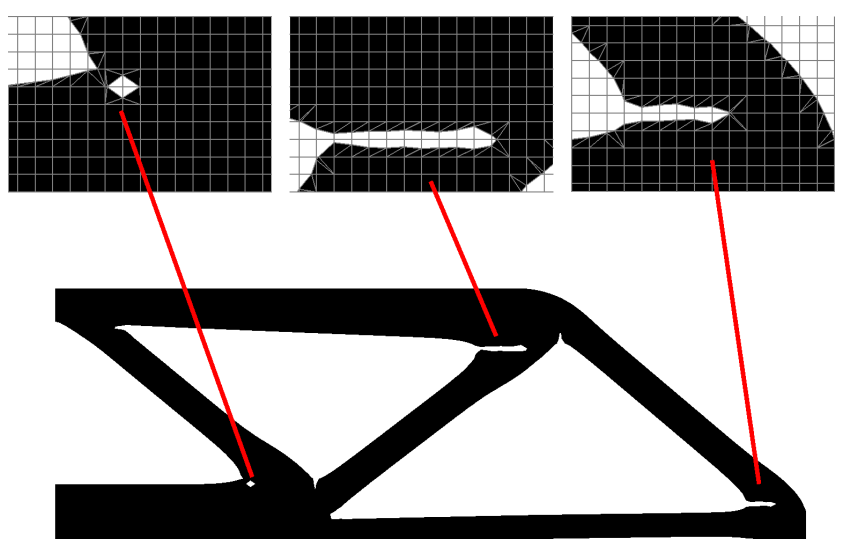

Fig. 14 An illustration of artificial stiffness gained by slender notches and a single node hole formed during optimization of the MBB beam when no robust formulation is used i.e. $\Delta \eta=0$ while maintaining the convolution filter with $R=10$ elements. Initial design was 11(c) and no hole generation was employed.

14. The design shows how the optimizer benefits from sub-element void features, and hence that a length scale is needed to mitigate the problem.

As described we rely on the robust formulation from the density method to ensure a length scale. As is the case for density methods, the resulting length scale depends on the filter radius, $R$, the steepness of the projection, $\beta$, the projection threshold $\eta$ and projection off-set $\Delta \eta$. Fig. 15 shows three optimized instances of the MBB beam using the initial design in Fig. 11(c) with an allowed volume fraction of $50 \%$ using a grid of $150 \times 50$ elements.

The optimized designs are shown by their contour lines representing the dilated (dashed), blue print (solid) and eroded (dotted), respectively. The minimum length scale possible is clearly visible and is, ideally, given by twice the distance from the blue print to the eroded realization. By inspection of the optimized designs it is seen that the smallest member size increases with growing $\Delta \eta$, which in turn leads to larger objective values. Compared to the density method, in which the steepness parameter is increased using a continuation scheme, we also observe that the chosen value of $\beta=12$ results in slight variations of the minimum length scale. However, if $\beta$ is increased further it will eventually lead to designs with undesired stair-casing interfaces as seen in density methods. A possible remedy for this minor shortcoming, is to apply the double filtering approach presented in Christiansen et al. (2015) which would alleviate the stair-casing while ensuring the exact prescribed length-scale. This is however deemed outside the scope of the presented work, since the presented examples clearly possess a length scale and effectively suppress sub-element size features. From the optimized MBB beams it is also seen that only the last example comes close to the actual minimum length scale, and we refer the reader to sections 5.1.6 and 5.2 for further investigations into the imposed length-scale.

Finally, it is also be noted that the length scale is only ensured for parts of the structure that is not in contact with the boundary of the design domain. This is a consequence of the symmetry condition of the applied filtering scheme and it could be cured by introducing padding to the design field as shown in e.g. (Clausen and Andreassen 2017).

\subsubsection{Mesh dependence}

An appealing quality of the cut element based optimization approach is the possibility to work with low resolution background meshes and still obtain designs with finely resolved interfaces. This is especially important since the numerical integration scheme as well as sensitivity analysis can be regarded as expensive compared to density based methods on a uniform and structured grid. To illustrate the effect of changing mesh resolution, the MBB design problem is solved for three different meshes. To make the comparison possible across the different meshes, the same physical filter radius is employed for all examples. The optimized designs are shown in Fig. 16 and are all obtained using the initial 


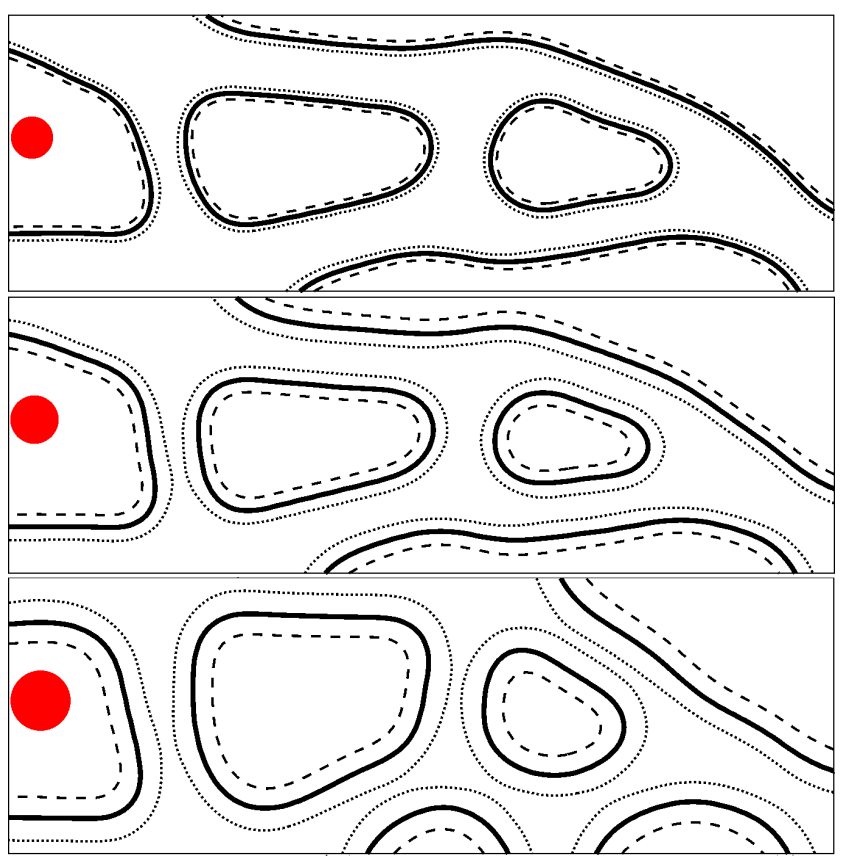

Fig. 15 Length scale illustration in optimized designs of the MBB beam on a mesh of $150 \times 50$ elements, a $50 \%$ volume fraction, a filter of $R=10$ elements with $\beta=12$ and $\Delta \eta=\{0.1,0.2,0.3\}$, respectively. The obtained objective values are $\Psi=\{226.7$ (164), 238.0 (322), 266.0 (583)\}, respectively, where the numbers in parenthesis denote the number of iterations needed to reach the stopping criteria. The lines corresponds to the dilated (dashed), blue print (solid line) and eroded (dotted) realization. The solid red circles correspond to the solid minimum feature size. All optimizations were started from the same initial design in Fig. 11(c).

configuration with three holes in Fig. 11(c) and without hole insertions.

Inspecting the three optimized designs in Fig. 16 reveals that all mesh resolutions yield well performing designs with similar objective values and final topologies. There are, however, small differences in the positioning of the junctions which is also reflected in the objective values that are within $1 \%$ of each other. Thus, it is clear that the proposed design approach is capable of obtaining mesh independent designs. Furthermore, the mesh dependency study confirms the assertion that the cut element optimization approach is well-suited for coarse mesh utilization, since there is little difference in the smoothness of the obtained designs across the different meshes.

\subsubsection{The cantilever beam}

Another classical minimum compliance example is the cantilever design problem sketched in Fig. 17. As for the MBB problem, several initial guesses have been considered as shown in Fig. 18 and they consist of a 6 by 4

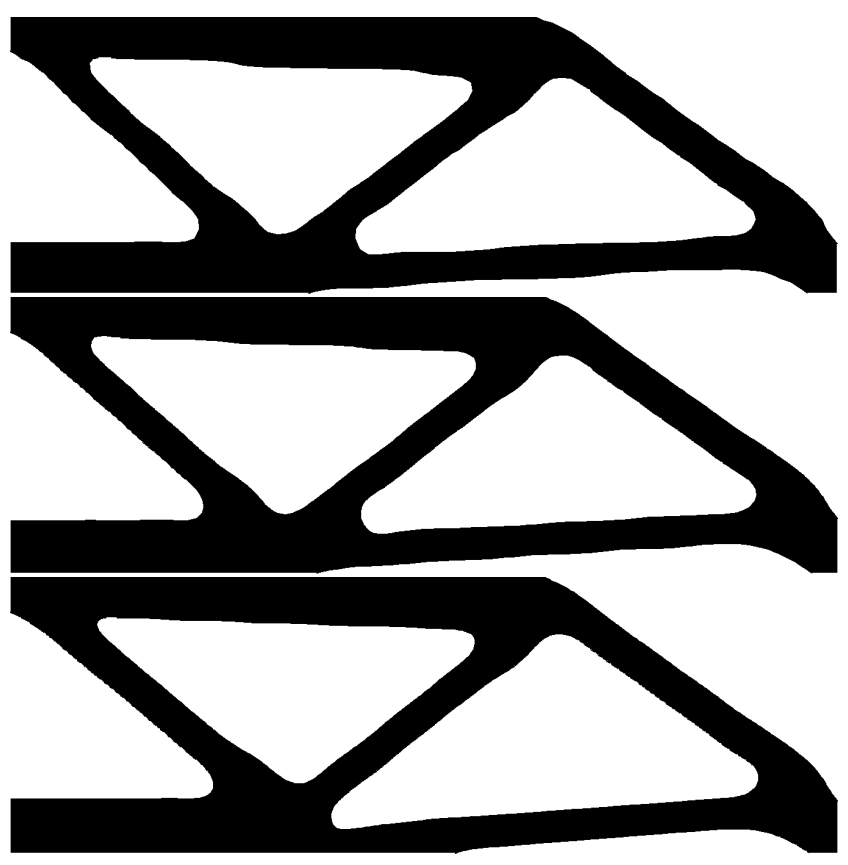

Fig. 16 Mesh independence study for the MBB beam. $90 \times 30 R=5,150 \times 50 R=8.3,300 \times 100 R=16.6$ $\Delta \eta=0.01$ volume fraction $40 \%$. The objective values are $\Psi=\{228.1,230.2,229.3\}$ and the results are obtained using the initial configuration with three holes in Fig. 11(c).

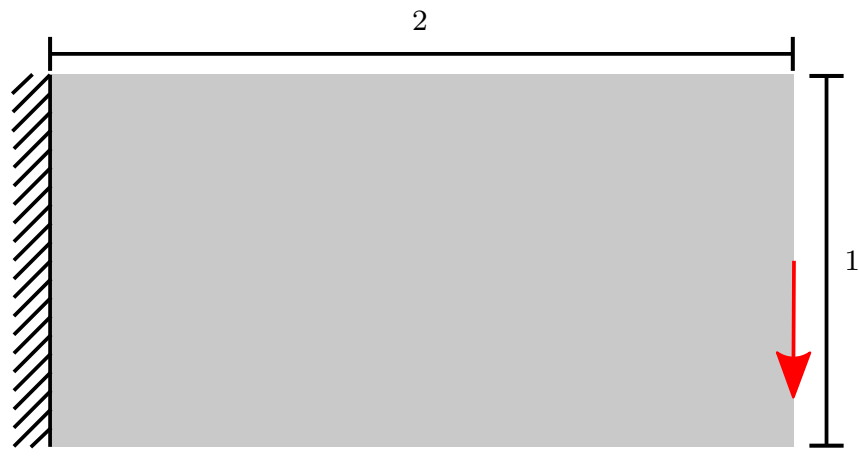

Fig. 17 Cantilever problem

hole configuration seen in Fig. 18(a), an almost solid design with cut edges along the free part of the exterior boundary shown in Fig. 18(b) as well as a fully solid configuration. As for the MBB beam the material parameters are set to $E=1$ and $\nu=0.3$.

First, the cantilever problem is solved without the hole insertion strategy using the initial guess in Fig. 18(a) with varying $\Delta \eta$ to verify the imposed length scale. The optimized design for a target volume of $40 \%$ and two different projection off-sets $\Delta \eta=0.01$ and $\Delta \eta=0.05$ are shown in Fig. 19. Inspecting the optimized designs reveal that the topology differs. This is contributed to the restriction on length scale imposed 
(a)

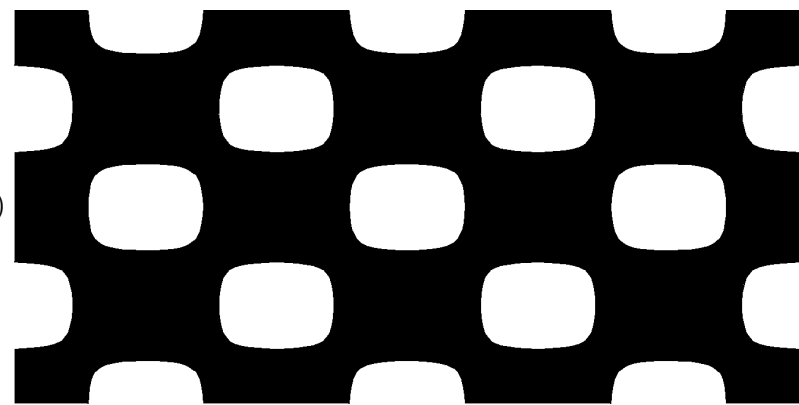

(b)

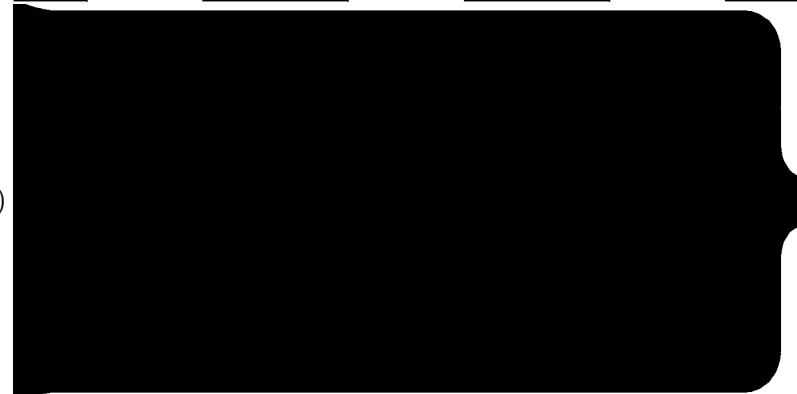

Fig. 18 Initial designs for cantilever problem. (a) a 6 by 4 hole configuration and (b) an almost solid design.

by the robust formulation. Also, due to the imposition of a larger length scale, the objective is slightly higher for the design with thicker bars. It is also seen that both designs display a small degree of wobbly edges and the frequency of these oscillations seems to relate to the filter radius. To examine this artifact further we perform a study in which the filter radius is varied.

Fig. 20 shows two optimized design obtained using a filter radius of $R=2$ and $R=7$, respectively. The remaining parameters are kept constant, i.e. a mesh of $100 \times 50$ elements, a target volume of $40 \%$ and $\Delta \eta=$ 0.01 . The fully solid initial design is used and the heuristic hole insertion strategy is enabled. The optimized design using a filter with $R=7$ display a smooth and crisp interface however, the relatively large filter radius and thus influence area when inserting holes have resulted in the development of an asymmetric topology. The breakdown of the symmetry is observed to be a consequence of the heuristic hole insertion scheme in combination with the chosen mesh resolution and filter radius. This is clearly not the case for $R=2$. Instead, the design with $R=2$ is polluted by a high degree of jagged edges, and hence does not display the desired smooth interface between solid and void, although the design remains symmetric. We note, however, that the small filter radius is sufficient regularization to avoid double-cuts and slender notches.

To further emphasize the need for regularization and the robust formulation, we present a cantilever design in Fig. 21 obtained with $\Delta \eta=0$ and a filter radius of $R=3$. The resulting design display several notches as

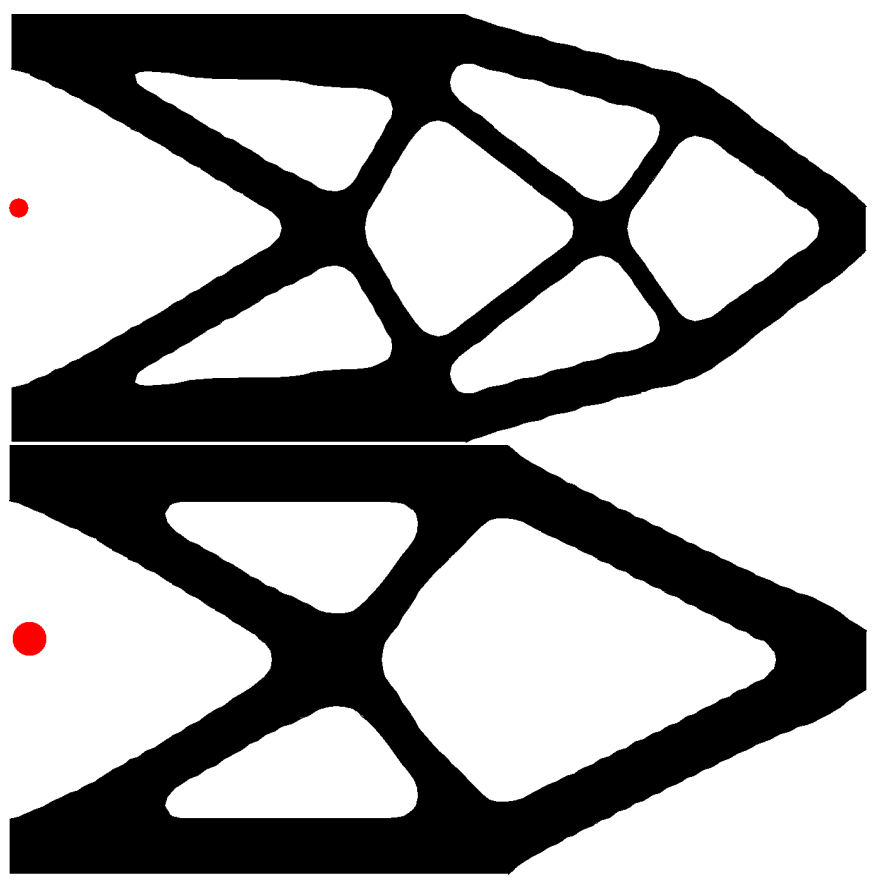

Fig. 19 Optimized design for the cantilever using $100 \times 50$ elements, $R=5$ nodes and $40 \%$ material. The initial guess is from Fig. 18(a) and the upper design is obtained using $\Delta \eta=0.01$ whereas the lower design uses $\Delta \eta=0.05$. The blueprint objective values are $\Psi=\{72.8,74.6\}$, respectively. The red circles illustrate the minimum feature size.

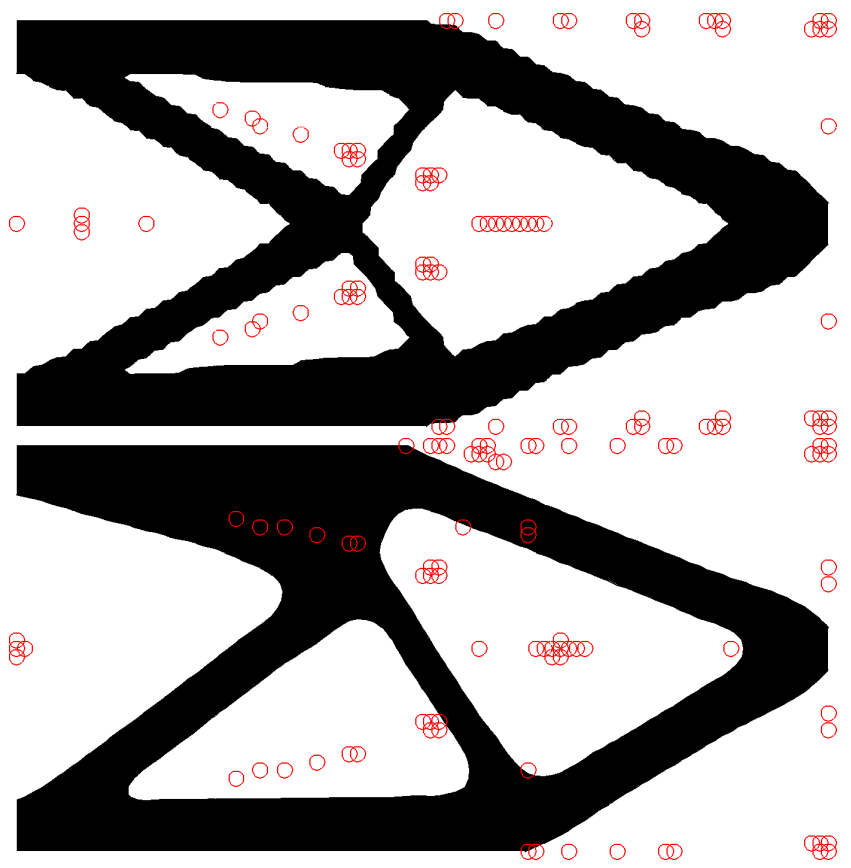

Fig. 20 Cantilever using two different filter radii on a mesh 100x50 elements, $\Delta \eta=0.01,40 \%$ material, a fully solid initial guess and with the hole insertion strategy. The top design uses $R=2$ and the bottom $R=7$ and have objectives $\Psi=$ $\{74.0 ; 75.7\}$, respectively. 
well as a double cut, and hence many of the numerical artifacts discussed in (Makhija and Maute 2014) in the context of an XFEM setting. However, contrary to the work of (Makhija and Maute 2014) we are able to avoid such features without adding enrichments to the finite element formulation. The double-cut is especially interesting since it is associated with an ambiguity in the extreme case of perfect arithmetic, i.e. when two design points are exactly $a$ and the other two are exactly $-a$. In this case, the center part of the element can be chosen to be either solid or void depending on the interpolated value of the level set in the element center. However, since all double cuts are associated with an artificially high stiffness, independent of the solid-void configuration, we choose to let the interior be void for all presented examples in this manuscript. This is deemed reasonable since the robust formulation effectively suppresses double cuts and notches, although these features may appear (and disappear) in the eroded design during the design process. We remark that some sub-element features could be suppressed by imposing a curvature constraint on the physical design field, however, a slender notch could still exist, just with a rounded tip. We also note that the design is locally asymmetric, which could be attributed to the triangulation of the cut elements being non-unique and hence that the placement of integration points may differ slightly for two symmetrically cut elements. This may eventually result in one being slightly stiffer than the other leading to the onset of an asymmetric design evolution. Finally, we remark that using a very large filter radius will lead to unintuitive designs with a loss of symmetry. Therefore it is important to emphasize that the filter radius should be chosen with care based on the chosen mesh resolution and $\Delta \eta$.

The cantilever example is completed with an investigation of the effect of varying target volume fractions, especially the low volume limit. This is relevant in the cut element setting due to its inability to represent intermediate densities which are likely to occur in density methods for low volume fractions. Using a fixed mesh of 100 by 50 elements, $R=5, \Delta \eta=0.01$ and the initial configuration in Fig. 18(b), the cantilever problem is solved for three different target volumes of $40 \%, 30 \%$ and $20 \%$. The optimized designs are shown in Fig. 22 and all demonstrate the proposed methods ability to produce designs with smooth material-void interfaces. An important observation is that going below $20 \%$ target volume on the chosen mesh resolution results in poorly performing designs and/or the breakdown of the optimization procedure due to non-connected load to support layouts. This means that although the proposed method is well suited for coarse meshes, the tar-

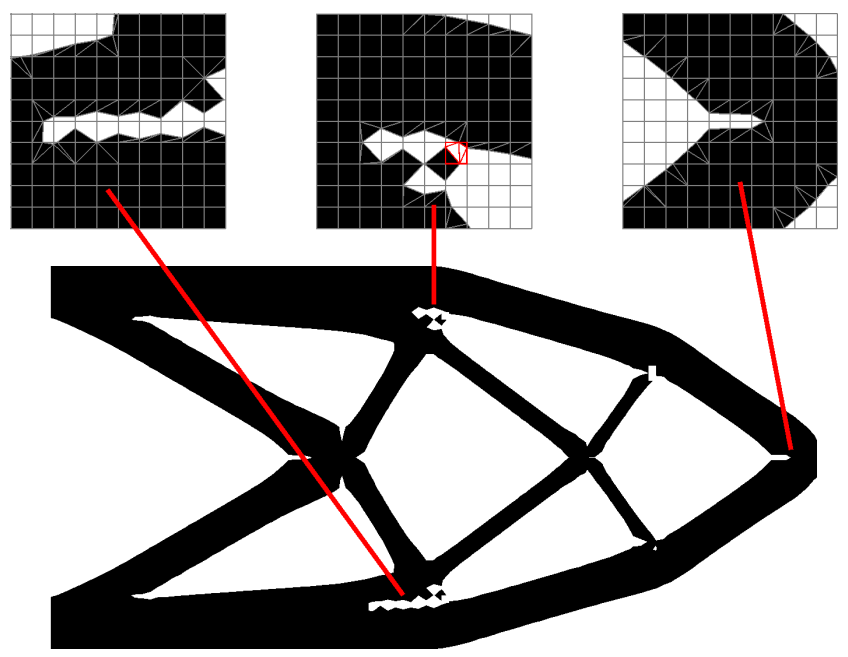

Fig. 21 An illustration of slender notches and double cuts formed during optimization of the cantilever if no robust formulation is used $\Delta \eta=0$ and $R=5$ elements. Initial structure Fig. 18(a) without hole generation. The three zooms show slender notches and double cuts that relies on artificial stiffness present in the cut-element model

get volume and the chosen mesh resolution must be correlated.

\subsubsection{Design of (1D) elastic bar}

The final minimum compliance problem is solely intended to demonstrate control of the minimum feature size. For this purpose the elastic bar design problem of Fig. 23 is used. This problem is especially well-suited for length-scale demonstration since it almost constitutes a 1D design problem, meaning that the optimal geometry is known in the form of a bar with uniform thickness connecting the support to the load. The design problem is solved on a mesh of $50 \times 50$ elements using the same parameters as already stated, with two exceptions being the MMA parameter $c=1000$ and a movelimit of $1 \%$. We aim for a target volume fraction of $10 \%$ for the blueprint design and solve the problem for two different filter radii and for varying projection offsets.

The optimized designs can be seen in Fig. 24 where the imposed minimum length scale is illustrated with solid red circles. First, we observe that only the design with $R=7$ and $\Delta \eta=0.1$ was capable of reaching the target volume. All other examples was hindered by the imposed minimum length scale in conjunction with the chosen MMA c parameter. That is, if the c parameter was increased significantly, the optimizer would simply ignore the objective function and the resulting designs would disconnect the load from the support. From the figure it is also evident that the eroded (dotted line) re- 


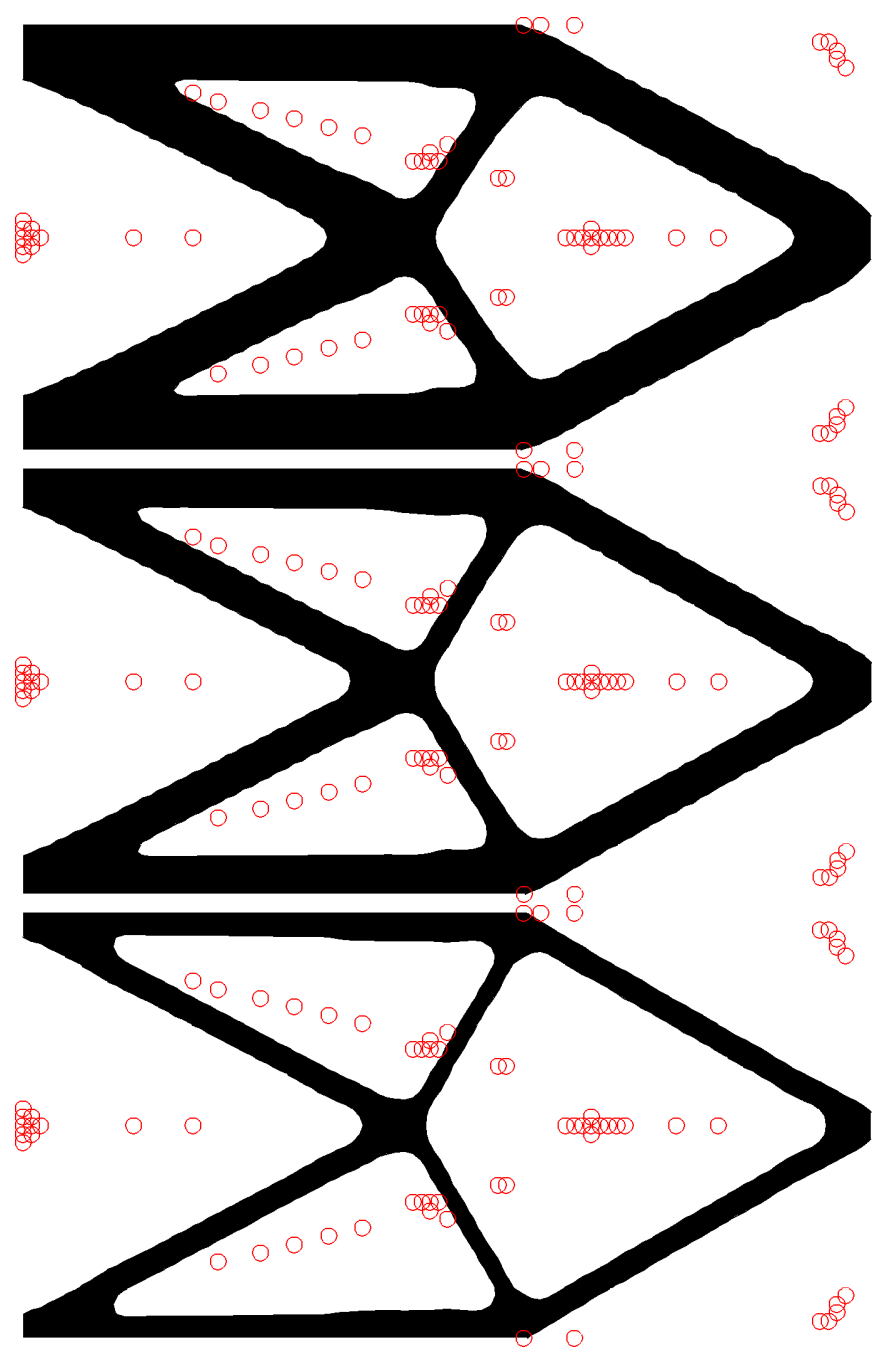

Fig. 22 Optimized cantilever designs for varying volume fractions, i.e. $40 \%, 30 \%$ and $20 \%$ using $\Delta \eta=0.01, R=5$ and the initial guess in Fig. 18(b). The resulting objectives are $\Psi=\{73.2,94.7,139.7\}$, respectively.

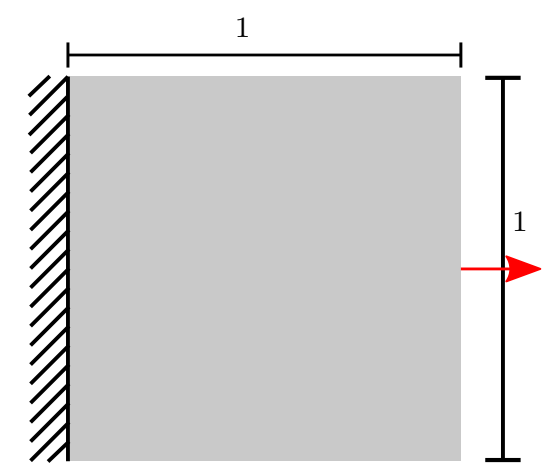

Fig. 23 Bar design problem mains constant for all presented designs, and hence that achieved minimum the length scale is defined as a combination of the chosen filter radius and the projection offset. Since the volume constraint is on the dilated design, the optimizer will try to push the dilated structure as close to the eroded as possible. This in turn ensures that the gradient of the level-set field near the interface attains its maximum slope i.e. a property defined by the filtering. This is visible from the plot of the various design fields shown in Fig. 25 of the $R=7, \Delta \eta=0.4$ design. However, a prescribed length scale can only be predicted (i.e. pre-computed based on the finding of (Wang et al. 2011)) if $\beta \rightarrow \infty$ which, for the proposed level set method, would result in non-smooth boundaries, i.e. stair casing. In summary, this small example study shows that the proposed design approach can be used to control the minimum feature size, analogous to that of density methods.

\subsection{Mechanism design - force inverter}

The numerical example section is concluded with the optimization of a compliant mechanism, more specifically the force inverter (Sigmund 1997). This design problem, which can be found sketched in Fig. 26, is more challenging for the cut element approach, and all other crisp interface level set methods for that matter, than the minimum compliance problem due to the following reason. Since the objective is to minimize the horizontal displacement of the output node, an obvious solution is to disconnect the output node from the input and the supports, and thus obtain zero output displacement. Furthermore, and in contrast to density based methods, it is close to impossible to make the structure re-connect if a disconnection occurs, since the cut element approach does not provide sensitivity information in the void region. This is not a pronounced issue for density methods, since material can grow out of the void region. In the cut element approach, one therefore have to ensure that the disconnection does not happen at any point during the design process. This can be achieved in several ways. For example, one can start with a configuration that already inverts the input force, use extremely small step lengths for the design update, reduce the contrast between solid and void, tune the input and output spring stiffnesses to name but a few. In this work the problem is overcome by adding an additional compliance constraint to the optimization formulation. The compliance constraint is intended to ensure a fully connected structure and for that purpose an auxiliary problem is introduced as shown in Fig. 27. The problem is almost identical to the model problem for the inverter itself, with the difference of an additional load 


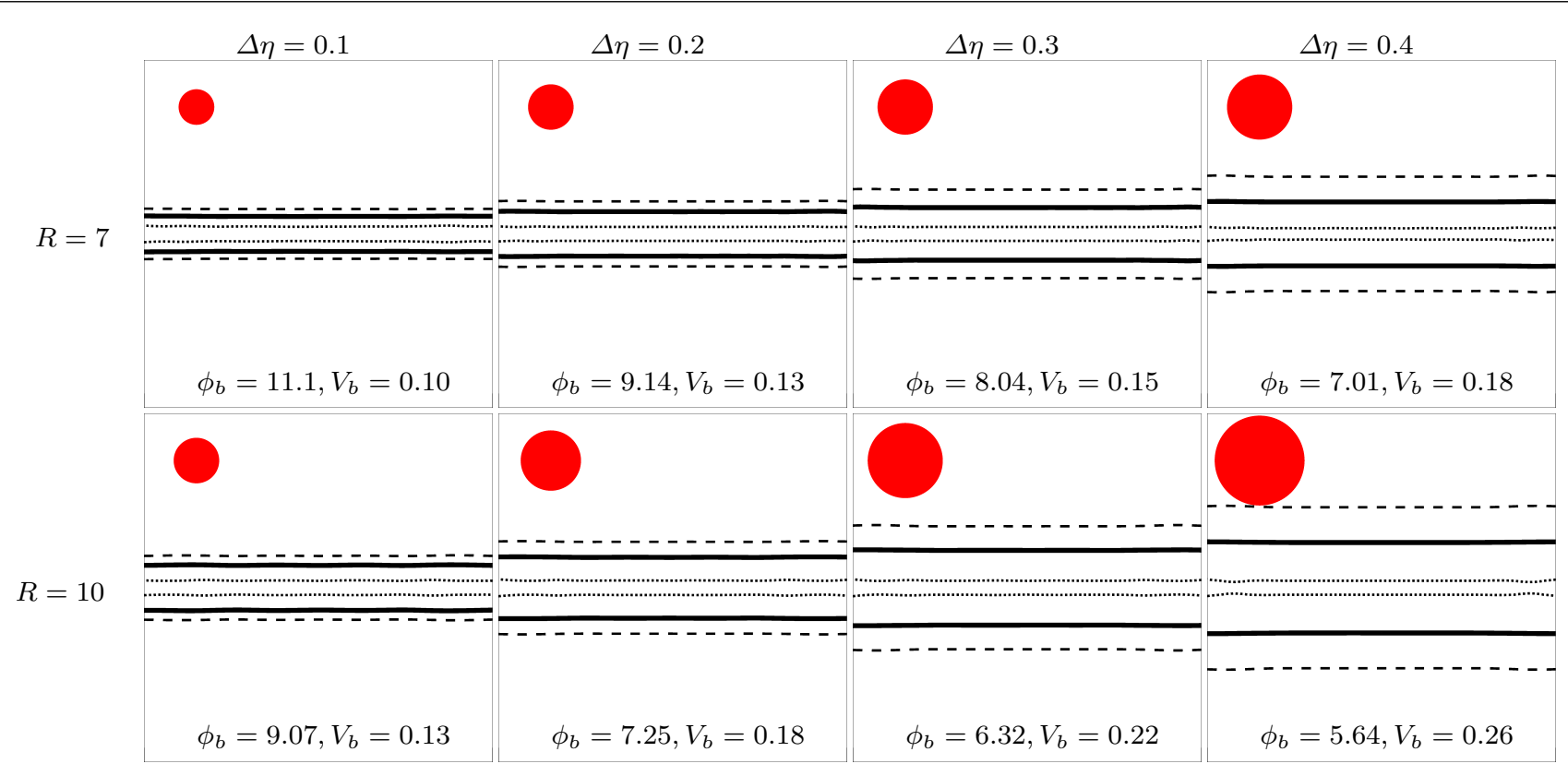

Fig. 24 Length scale demonstration for optimized designs of the (1D) bar design problem (Fig. 23 ) on a mesh of $50 \times 50$ elements and a $10 \%$ target volume fraction. The lines corresponds to the dilated (dashed), blue print (solid line) and eroded (dotted) realization and the red circles refers to the minimum length scale on the blueprint. All optimizations were started from a solid design with free horizontal boundaries.

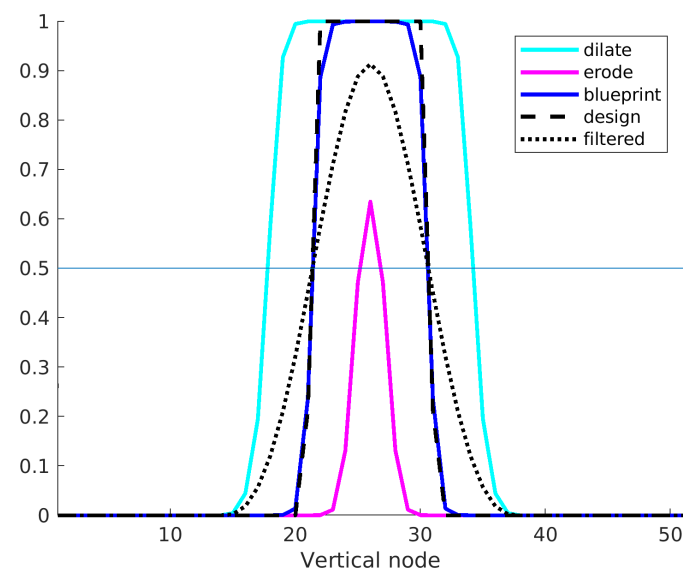

Fig. 25 Slice plot of the various design fields for the (1D) bar design problem with $R=7, \Delta \eta=0.4$.

on the output port. Hence, a bound on the compliance for the auxiliary problem will ensure that the two loads are connected to the support in the bottom left. Once a functioning mechanism is obtained, i.e. all realizations display negative output port displacements, the compliance constraint can be turned off, and thus the final part of the optimization process concerns the true inverter problem alone. We remark, that once the objective functions become negative, the sensitivity information will no longer point to a disconnection, as this will lead to an increase in objectives. It will, however, still try to make the connection as hinge-like as possible, which advocates the need for feature size control.

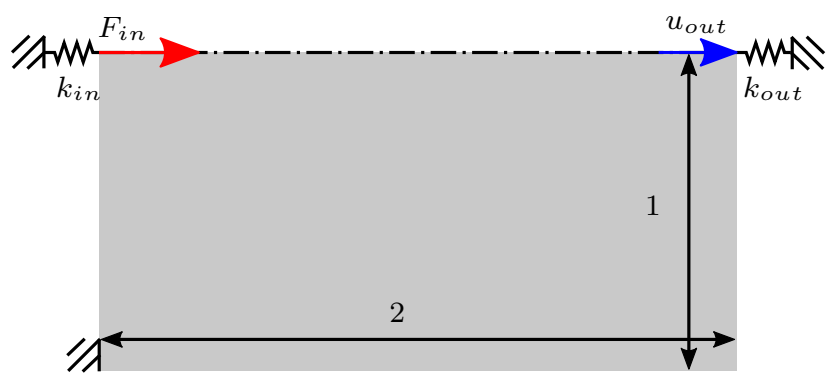

Fig. 26 Force inverter design problem. The material has stiffness $E=1$ and $\nu=0.3$ and the input and output springs are set to $k=0.1$. The input force is set to $F=1$.

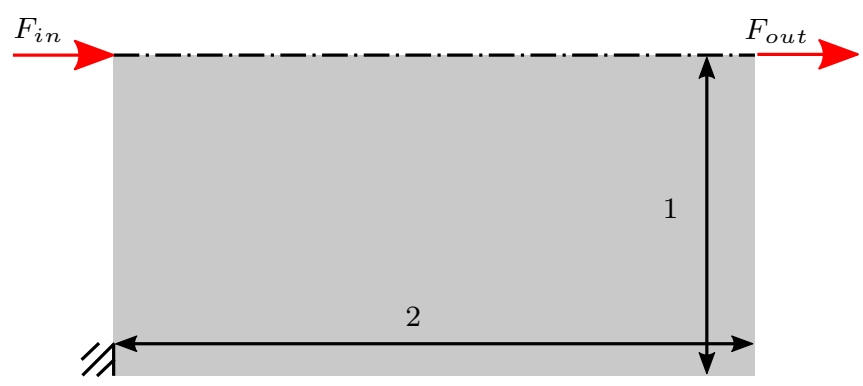

Fig. 27 Auxiliary compliance problem for the force inverter. Both forces are set to $F=1$.

The resulting optimization problem can be stated in full as the following mathematical program 


$$
\min _{\boldsymbol{s} \in \mathbb{R}^{n}}: \quad \Psi=\max _{k}\left\{\boldsymbol{L}^{T} \boldsymbol{u}^{k}\right\}, \quad k \in[e, b]
$$

subject to: $\mathbf{K}^{e}(\boldsymbol{s}) \mathbf{u}^{e}=\mathbf{f}$

$$
\begin{aligned}
& \mathbf{K}^{b}(\boldsymbol{s}) \mathbf{u}^{b}=\mathbf{f} \\
& \mathbf{K}_{a}^{e}(\boldsymbol{s}) \mathbf{u}_{a}^{e}=\mathbf{f}_{a} \\
& \int_{\Omega} \alpha^{e}(\boldsymbol{s}) \boldsymbol{\epsilon}\left(\boldsymbol{u}_{a}^{e}\right): \boldsymbol{\sigma}\left(\boldsymbol{u}_{a}^{e}\right) \mathrm{d} \Omega / c^{*}-1 \leq 0 \\
& \int_{\Omega} \alpha^{b}(\boldsymbol{s}) \mathrm{d} \Omega / V^{*}-1 \leq 0 \\
& 0 \leq s_{i} \leq 1, \quad \forall i
\end{aligned}
$$

where $e, b$ refers to the robust formulations eroded and blueprint design, respectively, and $\boldsymbol{L}$ is a vector of zeros with a single unitary non-zero placed at the position of the output dof. The auxiliary compliance constraint and accompanying finite element system are identified with subscript $a$ and the sensitivity of the constraints is identical to eq. (25) divided with $c^{*}$, which is a user defined value for the maximum allowable compliance. The non-smooth minimax problem is solved in a nested approach using a bound formulation, c.f. (Olhoff 1989). Contrary to the minimum compliance problem for which only a single finite element problem had to be solved per design cycle, this formulation requires the solution to three forward problems and two adjoints, one for each of the objective terms. The adjoint equations are as follows

$\mathbf{K}^{k} \boldsymbol{\lambda}^{k}=-\boldsymbol{L}, \quad k \in\{e, b\}$

and the sensitivities can subsequently be computed as

$\frac{\mathrm{d} \Psi^{k}}{\mathrm{~d} \phi_{i}}=\sum_{j}^{n}\left(\left[\boldsymbol{\lambda}_{j}^{k}\right]^{T} \frac{\partial \mathbf{k}_{j}^{k}}{\partial \phi_{i}} \mathbf{u}_{j}^{k}\right), \quad k \in\{e, b\}$

where the sum over $j$ refers to elements adjacent to node $i$. Finally, we remark that the volume constraint is imposed on the blue print design and that the dilated realization have been omitted, which means that the optimized result is only guarantied to be robust wrt. over-etching.

The design problem is first solved using the same parameters found to work well for the compliance minimization problems. For a mesh of $100 \times 50$ finite elements we employ a filter with radius $R=5$, a steepness parameter of $\beta=12, \Delta \eta=0.01$ and an initial configuration with 6 by 4 holes (c.f. bottom image in Fig. 28). The input and output springs are set to $k_{\text {in }}=k_{\text {out }}=0.1$, the target volume fraction is set to $30 \%$ and a target compliance of $c^{*}=70$ is chosen,

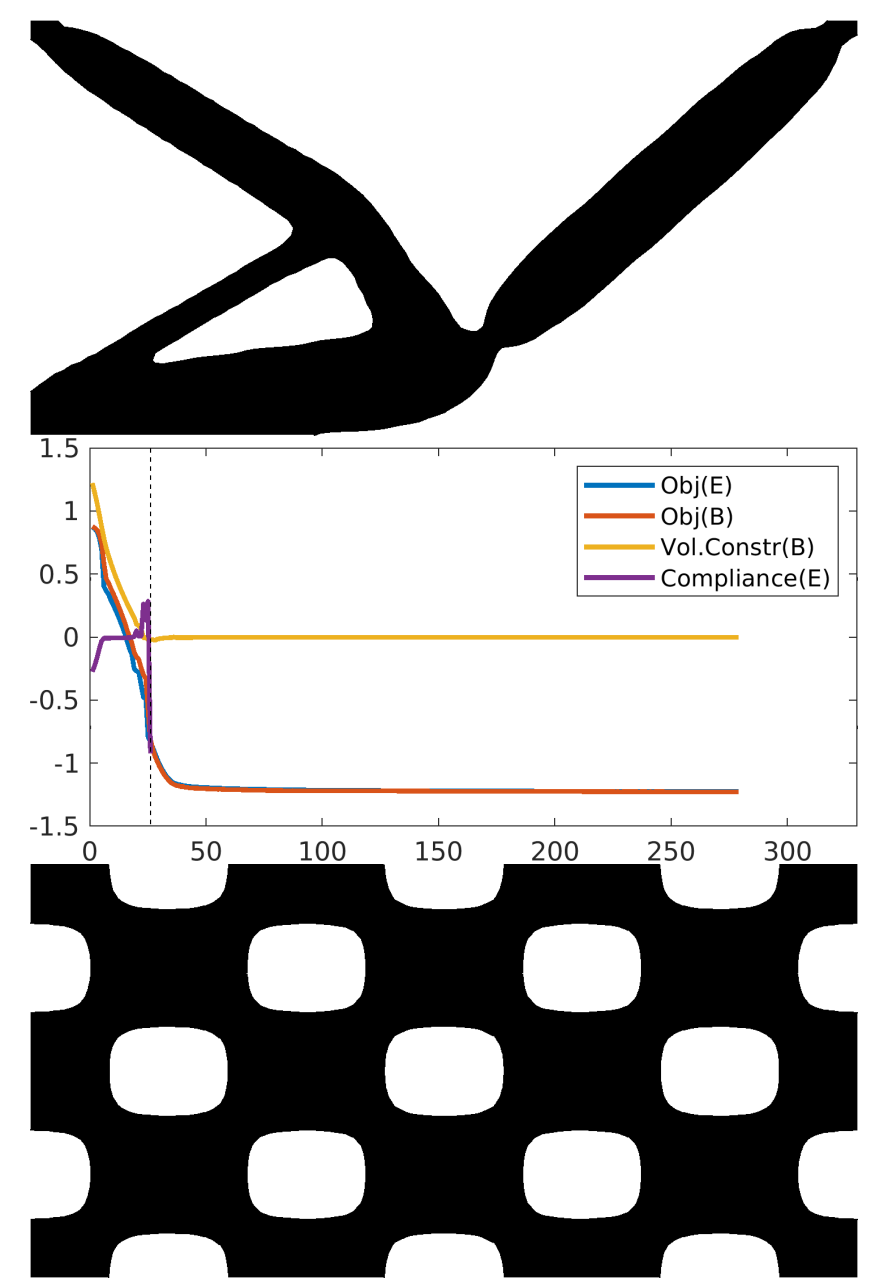

Fig. 28 Optimized inverter using $100 \times 50$ elements, $30 \%$ volume, $\Delta \eta=0.01, R=5$ elements, $\beta=12$ and a $6 b y 4$ initial design. The compliance target is set to $c^{*}=70$. The final objective values are $\Psi_{e}=-1.2247, \Psi_{b}=-1.2261$ for spring stiffnesses $k_{i n}=0.1$ and $k_{\text {out }}=0.1$, and the stopping criteria is reached after 279 cycles. The dashed vertical line indicate the point at which the compliance constraint is turned off.

which is slightly higher than the compliance of the initial configuration. The compliance constraint is turned off when $\Psi^{k}<-0.4$ for both realizations.

The resulting optimized force inverter can be seen in Fig. 28 which also shows the objective and constraint histories. From this it is seen that the stopping criteria is met after 279 cycles and that the compliance constraint is disabled after 26 iterations, indicated by the dashed vertical line. The design is seen to successfully invert the input force and result in a blueprint objective of $\Psi_{b}=-1.2261$. From the history plot it is clear that the compliance constraint remains inactive throughout most of the initial optimization process. It only becomes active during the cycles when the objective function goes from positive to negative and it is turned off immediately after reaching this point. 


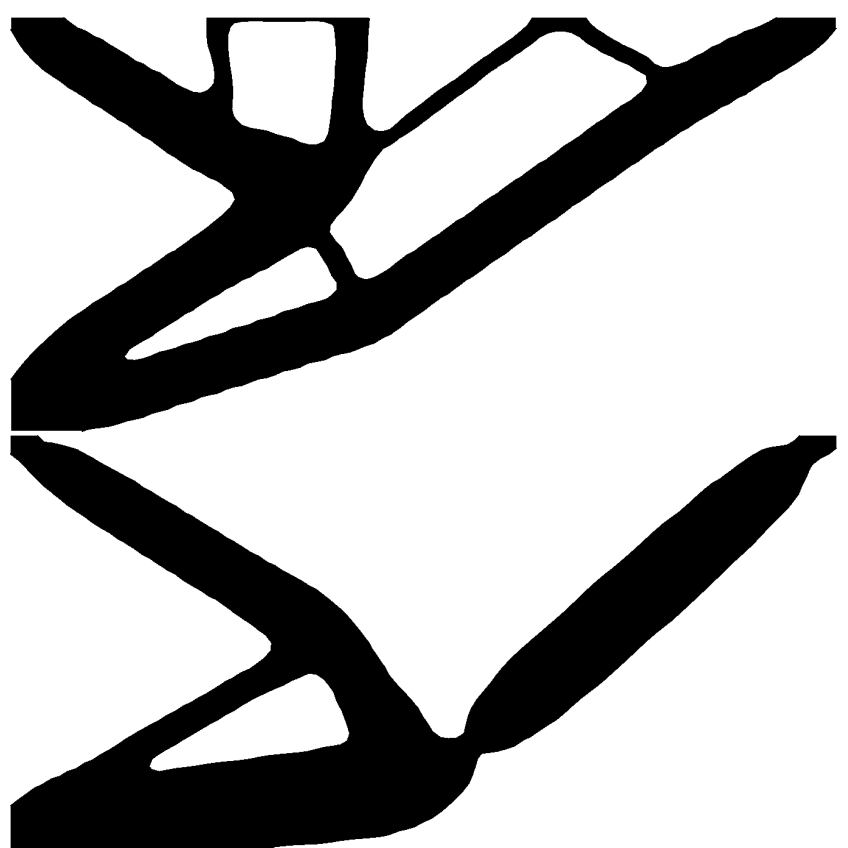

Fig. 29 Two force inverter designs obtained for varying compliance targets. The top design uses a $c^{*}=50$ while the bottom one is for $c^{*}=60$. The final objective values are $\Psi_{b}=0.0992$ and $\Psi_{b}=-1.2216$, respectively.

In order to investigate the importance of the choice of $c^{*}$, Fig. 29 shows two additional optimization results in which the target compliance is lowered to $c^{*}=50$ and $c^{*}=60$, respectively. From the top design it is seen that a too tight compliance constraint locks the optimizer and results in a design with a positive objective value, i.e. no force inversion. Since the objective(s) remain positive, the compliance constraint is found to be active throughout the optimization process and thereby hindering the force inversion. Loosening the compliance constraint leads to a well-performing design, with an objective value close to that seen in Fig. 28. This indicates that the auxiliary compliance constraint plays very little, if any, role wrt. solving the inverter problem once a feasible mechanism is obtained. If, on the other hand, the compliance target is increased substantially above 70 , the designs will disconnect from the output and the resulting objective value is zero. To summarize: a compliance constraint can be used to ensure a robust optimization procedure, but, at the same time the target value must be chosen appropriately in order to obtain good designs. Thus, a certain degree of parameter tuning is currently needed to solve mechanism design problems using the proposed methodology. It is desirable to device a robust heuristic for the choice of compliance target, however, this is deemed outside the scope of the presented manuscript.
Having established the use of a compliance constraint during the initial phase of the optimization process, the next step is to investigate the influence of the robust formulation on the optimized force inverters. This will reveal whether or not the compliance constraint alone is capable of achieving designs without numerical artifacts. Solving the the design problem in eq. (27) for $\Delta \eta=0$ leads to the design shown in Fig. 30(a). From the optimized design, and the two zoom inserts, it is clear that a force inverter is obtained, but also that undesired sub-element features have appeared near the hinge and at the top of the hole.

To investigate the length scale control the inverter problem is solved for $\Delta \eta=0.05$ and $\Delta \eta=0.10$ and the results are shown in Fig. 30(b-c). From the results one sees that the change in threshold parameters has the expected effect on both design and performance. That is, increasing $\Delta \eta$ leads to a larger minimum length scale and at the same time to a reduction in the objective function.

\section{Discussion and Conclusion}

This work considers a cut element shape and topology optimization methodology in which the design field representation and regularization is purely based on the density method. Especially, the proposed methodology adopts the so-called robust formulation from density methods in order to introduce a length scale. Based on a Matlab implementation, the methodology is demonstrated on several numerical examples covering both minimum compliance and compliant mechanism design problems. For the minimum compliance problem, a heuristic hole generation scheme is introduced to allow for true topology optimization, whereas an additional compliance constraint is introduced to ensure connected mechanism designs. We emphasize that the compliance constraint is only used to ensure connectivity while the objective values pass through the critical zero point, and that it is subsequently turned off. In summary, the authors argue that the proposed methodology can be viewed as a density based optimization method with an overlay of cut element opposed to the often used ersatz material FEM model. In that respect, this work demonstrates a clear overlap between density and the crisp interface level set method, and perhaps more importantly, this work provides the density community with an easily accessible access point to level set based optimization methods.

Despite its many attractive properties, the proposed method comes with several issues and shortcomings. The main issue is an inherent problem with exact interface methods and concerns the localized sensitivity 
(a)

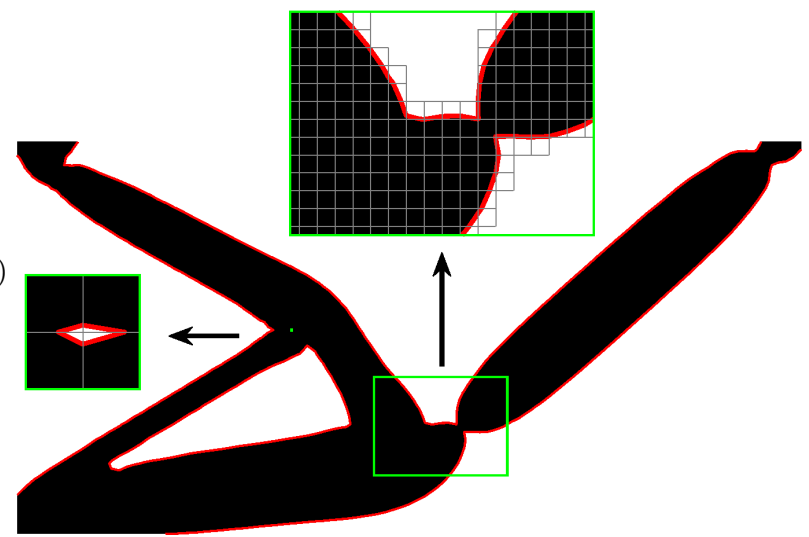

(b)

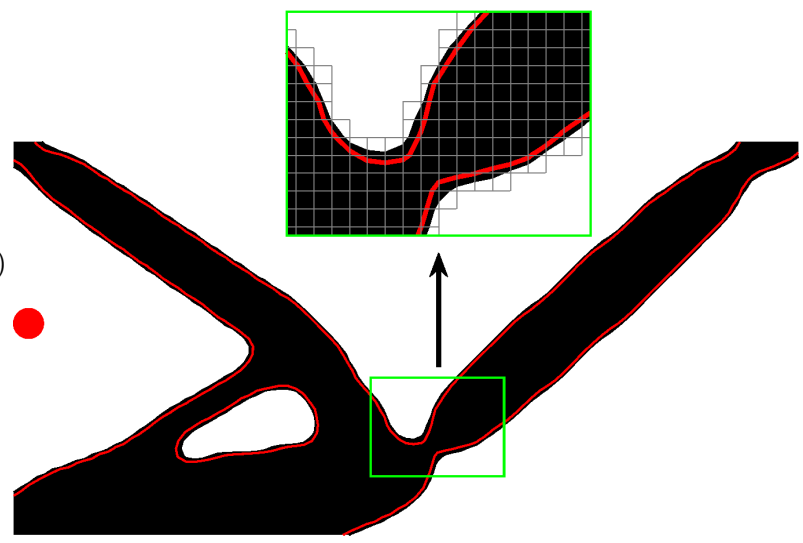

(c)

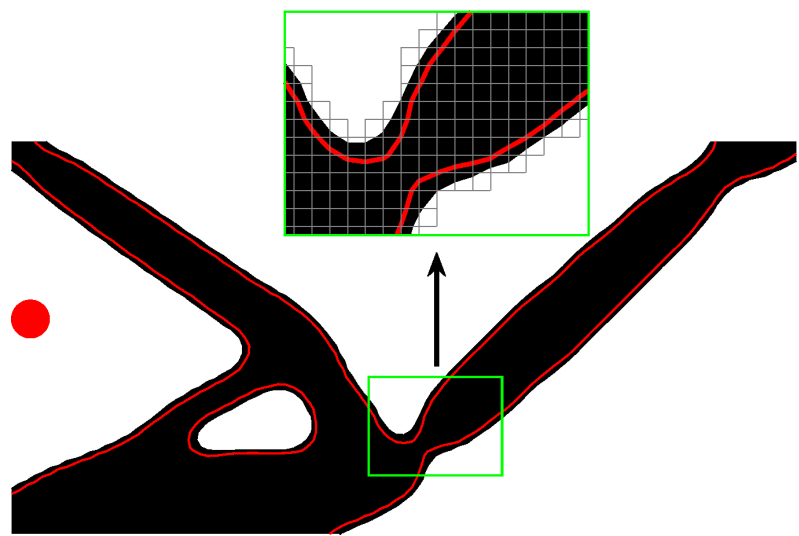

Fig. 30 The force inverter problem solved for varying $\Delta \eta$ using the same mesh and parameters as in Fig. 28. Plot (a) is for $\Delta \eta=0$ with $\Psi=-1.229$, (b) is for $\Delta \eta=0.05$ with $\Psi_{b}=-1.197$ and (c) is for $\Delta \eta=0.10$ with $\Psi_{b}=-1.168$. The red circles in the two bottom plots corresponds to the minimum length scale.

information. This is partially remedied by the image processing filters, but still means that only boundaries can move and hence, that no material can appear out of void. Another issue is due to the numerical properties of the cut element method which, similar to ersatz material models, does provide a stiffness in the void phase of a cut element. This can, and will, be utilized by the optimization algorithm, and hence a minimum length scale must be introduced to suppress this artifact. The method is also extremely sensitive to optimization parameter tuning, i.e. MMA parameters, level set bounds and maximum step length for the design update.

Similar to other shape optimization approaches, the optimized design is highly dependent on the chosen initial configuration. Compared to density methods this is mainly due to the difficulties in automatically generating an initial configuration fulfilling the target volume constraint, especially for low volume fractions.

Although not explicitly addressed in the numerical examples, the run time for the numerical examples are also far greater than that used by density method in e.g. the 99-line or 88-line Matlab codes. This is also evident in the methods coding complexity. That is, the accompanying code is several hundred lines of code mainly due to the cut element modelling and the slightly more involved sensitivity analysis.

A consistent, and probably the most important, observation from the numerical experiments is that the best results are obtained when using a density optimized design as the starting guess. That is, the cut element method is an excellent post processing tool for the presented example problems, but will display its real strength for optimization problems with crucial interface conditions such as elastic contact (Lawry and Maute 2018), fluid-structure interaction (Jenkins and Maute 2015) and multi-material structures (Faure et al. 2017) to name a few problems currently solved using XFEM.

There are several obvious future research directions based on the proposed methodology and available code. Besides interface dominated problems, e.g. pressure loading and multiphysics problems, this include a rewrite of the code using a lower level language than Matlab, e.g. $\mathrm{C} / \mathrm{C}++$ or Fortran. This may allow the element integration, the finite element assembly and the sensitivity calculation to reach speeds similar to those of the density method. Extending the code to $3 \mathrm{D}$ is also straightforward, although the quadrilaterals and triangles should be exchanged with hexagonals and tetrahedrals, respectively. Since the cut element optimization framework is based on a logical structured mesh, it is extremely well suited for parallel computing, as it does not require remeshing or renumbering of the dof during the optimization process. Therefore, the TopOpt in PETSc framework presented in Aage et al. (2015) can with some modifications be used to perform cut element based structural optimization. Regarding length scale imposition, the presented work does to some extent ensure this in the optimized design. However, in order to obtain a true length scale, the projection parameter must be set very high, which will result in designs with jagged edges. Therefore, it would be interesting 
to further explore the density based robust formulation using the double filtering approach, c.f. (Christiansen et al. 2015). Also, a hole generation scheme for the mechanism design problems is an interesting path to follow. Finally, it is clear that the proposed method lacks the robustness and simplicity of the standard density method. Thus, it would be highly desirable if one could reduce the complexity of the cut element optimization method and at the same time increase its robustness.

\section{Reproduction of results}

The authors have stored all data related to the presented examples and ensured that all relevant parameters, including the cut element method, are stated clearly throughout the manuscript. Furthermore, the base Matlab code capable of solving the minimum compliance problem is freely available from www.topopt.dtu.dk. However, we note that the user could MEX-compile some of the functions to achieve a reasonable performance compared to e.g. the 88-line density code.

Acknowledgements The authors would like to thank the Villum Foundation for the funding of the Villum Investigator grant through the InnoTop project. Furthermore, the members of the TopOpt group at the Technical University of Denmark should be acknowledged for fruitful discussions and so should associate senior lecturer Eddie Wadbro, Umeå University, Sweden and MSc Reinier Giele, TU Delft. We would also like to thank the anonymous reviewers for their constructive feedback.

\section{Conflict of interest}

On behalf of all authors, the corresponding author states that there is no conflict of interest.

\section{References}

Aage N, Nobel-Jørgensen M, Andreasen CS, Sigmund O (2013) Interactive topology optimization on hand-held devices. Structural and Multidisciplinary Optimization 47(1):1-6, DOI 10.1007/s00158-012-0827-z

Aage N, Andreassen E, Stefanov Lazarov B (2015) Topology optimization using PETSc: An easy-to-use, fully parallel, open source topology optimization framework. Structural and Multidisciplinary Optimization 51:565-572, DOI 10. 1007/s00158-014-1157-0

Aage N, Andreassen E, Lazarov BS, Sigmund O (2017) Gigavoxel computational morphogenesis for structural design. Nature 550(7674):84-86, DOI 10.1038/nature23911

Allaire G, Jouve F, Toader AM (2004) Structural optimization using sensitivity analysis and a level-set method. Journal of Computational Physics 194(1):363-393, DOI 10.1016/j.jcp.2003.09.032
Allaire G, Dapogny C, Frey P (2013) A mesh evolution algorithm based on the level set method for geometry and topology optimization. Structural and Multidisciplinary Optimization 48(4):711-715, DOI 10.1007/ s00158-013-0929-2

Allaire G, Dapogny C, Frey P (2014) Shape optimization with a level set based mesh evolution method. Computer Methods in Applied Mechanics and Engineering 282:2253, DOI 10.1016/j.cma.2014.08.028

Amir O, Aage N, Lazarov BS (2014) On multigrid-CG for efficient topology optimization. Structural and Multidisciplinary Optimization 49(5):815-829, DOI 10.1007/ s00158-013-1015-5

Andreasen CS, Gersborg AR, Sigmund O (2009) Topology optimization of microfluidic mixers. International Journal for Numerical Methods in Fluids 61(5):498-513, DOI 10. 1002/fld.1964

Andreassen E, Clausen A, Schevenels M, Lazarov BS, Sigmund O (2011) Efficient topology optimization in MATLAB using 88 lines of code. Structural and Multidisciplinary Optimization 43:1-16, DOI 10.1007/ s00158-010-0594-7

Beghini LL, Beghini A, Katz N, Baker WF, Paulino GH (2014) Connecting architecture and engineering through structural topology optimization. Engineering Structures 59:716-726, DOI 10.1016/j.engstruct.2013.10.032

Belytschko T, Parimi C, Moës N, Sukumar N, Usui S (2003) Structured extended finite element methods for solids defined by implicit surfaces. International Journal for Numerical Methods in Engineering 56(4):609-635, DOI $10.1002 /$ nme. 686

Bendsøe MP (1989) Optimal shape design as a material distribution problem. Structural Optimization 1(4):193-202, DOI 10.1007/BF01650949

Bendsøe MP, Kikuchi N (1988) Generating optimal topologies in structural design using a homogenisation method. Computer Methods in Applied Mechanics and Engineering 71(2):197-224, DOI 10.1016/0045-7825(88)90086-2

Bendsøe MP, Sigmund O (2004) Topology Optimization. Engineering online library, Springer Berlin Heidelberg, Berlin, Heidelberg, DOI 10.1007/978-3-662-05086-6

Bernland A, Wadbro E, Berggren M (2018) Acoustic shape optimization using cut finite elements. International Journal for Numerical Methods in Engineering 113(3):432449, DOI 10.1002/nme.5621

Borrvall T, Petersson J (2003) Topology optimization of fluids in Stokes flow. International Journal for Numerical Methods in Fluids 41(1):77-107, DOI 10.1002/fld.426

Bourdin B (2001) Filters in topology optimization. International Journal for Numerical Methods in Engineering 50(December 1999):2143-2158, DOI 10.1002/nme.116

Burman E, Claus S, Hansbo P, Larson MG, Massing A (2014) CutFEM: Discretizing geometry and partial differential equations. International Journal for Numerical Methods in Engineering 104:472-501, DOI 10.1002/nme.4823, 1201.4903

Burman E, Elfverson D, Hansbo P, Larson MG, Larsson K (2018) Shape optimization using the cut finite element method. Computer Methods in Applied Mechanics and Engineering 328:242-261, DOI 10.1016/j.cma.2017. $09.005,1611.05673$

Burman E, Elfverson D, Hansbo P, Larson MG, Larsson K (2019) Cut topology optimization for linear elasticity with coupling to parametric nondesign domain regions. Computer Methods in Applied Mechanics and Engineering 350:462-479, DOI 10.1016/j.cma.2019.03.016, 
1809.07503

Chen S, Chen W (2011) A new level-set based approach to shape and topology optimization under geometric uncertainty. Structural and Multidisciplinary Optimization 44(1):1-18, DOI 10.1007/s00158-011-0660-9

Chen S, Wang MY, Liu AQ (2008) Shape feature control in structural topology optimization. Computer-Aided Design 40(9):951-962, DOI 10.1016/j.cad.2008.07.004

Chen S, Chen W, Lee S (2010) Level set based robust shape and topology optimization under random field uncertainties. Structural and Multidisciplinary Optimization 41(4):507-524, DOI 10.1007/s00158-009-0449-2

Choi KK, Kim NH (2005) Structural Sensitivity Analysis and Optimization 1: Linear Systems. Mechanical Engineering Series, Springer, DOI 10.1007/b138709

Christiansen AN, Nobel-Jørgensen M, Aage N, Sigmund O, Bærentzen JA (2014) Topology optimization using an explicit interface representation. Structural and Multidisciplinary Optimization 49(3):387-399, DOI 10.1007/ s00158-013-0983-9

Christiansen RE, Sigmund O (2016) Experimental validation of systematically designed acoustic hyperbolic meta material slab exhibiting negative refraction. Applied Physics Letters 109(10), DOI 10.1063/1.4962441

Christiansen RE, Lazarov BS, Jensen JS, Sigmund O (2015) Creating geometrically robust designs for highly sensitive problems using topology optimization: Acoustic cavity design. Structural and Multidisciplinary Optimization 52(4):737-754, DOI 10.1007/s00158-015-1265-5

Clausen A, Andreassen E (2017) On filter boundary conditions in topology optimization. Structural and Multidisciplinary Optimization 56(5):1147-1155, DOI 10.1007/ s00158-017-1709-1

Cook RD, Malkus DS, Plesha ME, Witt RJW (2002) Concept and Applications of Finite Element Analysis

Dapogny C, Faure A, Michailidis G, Allaire G, Couvelas A, Estevez R (2017) Geometric constraints for shape and topology optimization in architectural design. Computational Mechanics 59(6):1-33, DOI 10.1007/ s00466-017-1383-6

Daux C, Moës N, Dolbow J, Sukumar N, Belytschko T (2000) Arbitrary branched and intersecting cracks with the extended finite element method. International Journal for Numerical Methods in Engineering 48(12):1741-1760, DOI 10.1002/1097-0207(20000830)48: 12〈1741::AID-NME956〉3.0.CO;2-L

Dilgen SB, Dilgen CB, Fuhrman DR, Sigmund O, Lazarov BS (2018) Density based topology optimization of turbulent flow heat transfer systems. Structural and Multidisciplinary Optimization 57(5):1905-1918, DOI 10.1007/ s00158-018-1967-6

Dühring MB, Jensen JS, Sigmund O (2008) Acoustic design by topology optimization. Journal of Sound and Vibration 317:557-575, DOI 10.1016/j.jsv.2008.03.042

Düster A, Parvizian J, Yang Z, Rank E (2008) The finite cell method for three-dimensional problems of solid mechanics. Computer Methods in Applied Mechanics and Engineering 197(45):3768-3782, DOI 10.1016/j.cma.2008.02. 036

Eschenauer HA, Kobelev VV, Schumacher A (1994) Bubble method for topology and shape optimization of structures. Structural Optimization 8(1):42-51, DOI 10.1007/ BF01742933

Evgrafov A, Rupp CJ, Maute K, Dunn ML (2008) Large-scale parallel topology optimization using a dual-primal substructuring solver. Structural and Multidisciplinary Op- timization 36:329-345, DOI 10.1007/s00158-007-0190-7

Faure A, Michailidis G, Parry G, Vermaak N, Estevez R (2017) Design of thermoelastic multi-material structures with graded interfaces using topology optimization. Structural and Multidisciplinary Optimization pp 1-15, DOI 10.1007/s00158-017-1688-2

Feijóo Ra, Novotny Aa, Taroco E, Padra C (2003) The topological derivative for the poisson's problem. Mathematical Models and Methods in Applied Sciences 13(12):18251844, DOI 10.1142/S0218202503003136

Guest JK, Prévost JH, Belytschko T (2004) Achieving minimum length scale in topology optimization using nodal design variables and projection functions. International Journal for Numerical Methods in Engineering 61(September 2003):238-254, DOI 10.1002/nme.1064

Hansbo A, Hansbo P (2002) An unfitted finite element method for elliptic interface problems. Comput Methods Appl Mech Engrg 191:5537 - 5552, DOI 10.1016/ S0045-7825(02)00524-8

Jansen M (2018) Explicit level set and density methods for topology optimization with equivalent minimum length scale constraints. Structural and Multidisciplinary Optimization 59:1775-1788, DOI 10.1007/s00158-018-2162-5

Jenkins N, Maute K (2015) Level set topology optimization of stationary fluid-structure interaction problems. Structural and Multidisciplinary Optimization 52(1):179-195, DOI 10.1007/s00158-015-1229-9

Kreissl S, Maute K (2012) Levelset based fluid topology optimization using the extended finite element method. Structural and Multidisciplinary Optimization 46(3):311-326, DOI 10.1007/s00158-012-0782-8

Larsen U, Signund O, Bouwsta S (1997) Design and fabrication of compliant micromechanisms and structures with negative Poisson's ratio. Journal of Microelectromechanical Systems 6(2):99-106, DOI 10.1109/84.585787

Lawry M, Maute K (2018) Level set shape and topology optimization of finite strain bilateral contact problems. International Journal for Numerical Methods in Engineering 113(8):1340-1369, DOI 10.1002/nme.5582

Lazarov BS, Wang F, Sigmund O (2016) Length scale and manufacturability in density-based topology optimization. Archive of Applied Mechanics 86(1-2):189-218, DOI 10.1007/s00419-015-1106-4

Lorensen WE, Cline HE (1987) Marching cubes: A high resolution 3D surface construction algorithm. In: Proceedings of the 14th annual conference on Computer graphics and interactive techniques - SIGGRAPH '87, ACM Press, New York, New York, USA, vol 21, pp 163-169, DOI 10.1145/37401.37422

Lundgaard C, Alexandersen J, Zhou M, Andreasen CS, Sigmund O (2018) Revisiting density-based topology optimization for fluid-structure-interaction problems. Structural and Multidisciplinary Optimization 58(February):1-27, DOI 10.1007/s00158-018-1940-4

Makhija D, Maute K (2014) Numerical instabilities in level set topology optimization with the extended finite element method. Structural and Multidisciplinary Optimization 49(2):185-197, DOI 10.1007/s00158-013-0982-x

Nitsche J (1971) Über ein Variationsprinzip zur Lösung von Dirichlet-Problemen bei Verwendung von Teilräumen, die keinen Randbedingungen unterworfen sind. Abhandlungen aus dem Mathematischen Seminar der Universität Hamburg 36(1):9-15, DOI 10.1007/BF02995904

Nobel-Jørgensen M, Malmgren-Hansen D, Bærentzen JA, Sigmund O, Aage N (2016) Improving topology optimization intuition through games. Structural and 
Multidisciplinary Optimization pp 1-7, DOI 10.1007/ s00158-016-1443-0

Olhoff N (1989) Multicriterion structural optimization via bound formulation and mathematical programming. Structural Optimization 1(1):11-17, DOI 10.1007/ BF01743805

Osher S, Sethian JA (1988) Fronts propagating with curvature-dependent speed: Algorithms based on Hamilton-Jacobi formulations. Journal of Computational Physics 79(1):12-49, DOI 10.1016/0021-9991(88)90002-2, 9809069v1

Querin O, Steven G, Xie Y (1998) Evolutionary structural optimisation (ESO) using a bidirectional algorithm. Engineering Computations 15(8):1031-1048, DOI 10.1108/ 02644409810244129

Schevenels M, Lazarov BS, Sigmund O (2011) Robust topology optimization accounting for spatially varying manufacturing errors. Computer Methods in Applied Mechanics and Engineering 200(49-52):3613-3627, DOI 10.1016/ j.cma.2011.08.006

Sethian J, Wiegmann A (2000) Structural Boundary Design via Level Set and Immersed Interface Methods. Journal of Computational Physics 163(2):489-528, DOI 10.1006/ jcph.2000.6581, arXiv:1011.1669v3

Sharma A, Maute K (2017) Stress-based topology optimization using spatial gradient stabilized XFEM. Structural and Multidisciplinary Optimization pp 1-22, DOI 10.1007/s00158-017-1833-y

Sharma A, Villanueva H, Maute K (2017) On shape sensitivities with heaviside-enriched XFEM. Structural and Multidisciplinary Optimization 55(2):385-408, DOI 10.1007/ s00158-016-1640-x

Sigmund O (1997) On the Design of Compliant Mechanisms Using Topology Optimization. Mech Struct \& Mach 25(4):493-524, DOI 10.1080/08905459708945415

Sigmund O (2001a) A 99 line topology optimization code written in matlab. Structural and Multidisciplinary Optimization 21(1999):120-127, DOI 10.1007/s001580050176

Sigmund O (2001b) Design of multiphysics actuators using topology optimization - Part I: One-Material structures. Computer Methods in Applied Mechanics and Engineering 190(49-50):6605-6627

Sigmund O (2009) Manufacturing tolerant topology optimization. Acta Mechanica Sinica 25(2):227-239, DOI $10.1007 / \mathrm{s} 10409-009-0240-\mathrm{z}$

Sigmund O (2011) On the usefulness of non-gradient approaches in topology optimization. Structural and Multidisciplinary Optimization 43:589-596, DOI 10.1007/ s00158-011-0638-7

Sigmund O, Clausen PM (2007) Topology optimization using a mixed formulation: An alternative way to solve pressure load problems. Computer Methods in Applied Mechanics and Engineering 196:1874-1889, DOI 10.1016/j.cma. 2006.09.021

Sigmund O, Maute K (2013) Topology optimization approaches. Structural and Multidisciplinary Optimization 48(6):1031-1055, DOI 10.1007/s00158-013-0978-6

Sigmund O, Torquato S (1996) Composites with extremal thermal expansion coefficients. Applied Physics Letters 69(21):3203-3205, DOI 10.1063/1.117961

Sokolowski J, Zochowski A (1999) On the Topological Derivative in Shape Optimization. SIAM Journal on Control and Optimization 37(4):1251-1272, DOI 10.1137/ S0363012997323230

Svanberg K (1987) The method of moving asymptotes - a new method for structural optimization. International
Journal for Numerical Methods in Engineering 24(June 1986):359-373, DOI 10.1002/nme.1620240207

Van Dijk NP, Maute K, Langelaar M, Van Keulen F (2013) Level-set methods for structural topology optimization: A review. Structural and Multidisciplinary Optimization 48(3):437-472, DOI 10.1007/s00158-013-0912-y

Van Miegroet L, Duysinx P (2007) Stress concentration minimization of 2D filets using X-FEM and level set description. Structural and Multidisciplinary Optimization 33(45):425-438, DOI 10.1007/s00158-006-0091-1

Villanueva CH, Maute K (2017) CutFEM topology optimization of 3D laminar incompressible flow problems. Computer Methods in Applied Mechanics and Engineering 320:444-473, DOI 10.1016/j.cma.2017.03.007, 1702. 02473

Wang F, Jensen JS, Sigmund O (2011) Robust topology optimization of photonic crystal waveguides with tailored dispersion properties. Journal of the Optical Society of America B 28(3):387, DOI 10.1364/JOSAB.28.000387

Wang MY, Wang X, Guo D (2003) A level set method for structural topology optimization. Computer Methods in Applied Mechanics and Engineering 192(1):227-246, DOI 10.1016/S0045-7825(02)00559-5

Wang S, Wang MY (2006) Radial basis functions and level set method for structural topology optimization. International Journal for Numerical Methods in Engineering 65(12):2060-2090, DOI 10.1002/nme.1536

Wei P, Wang MY, Xing X (2010) A study on X-FEM in continuum structural optimization using a level set model Computer-Aided Design 42(8):708-719, DOI 10.1016/j. cad.2009.12.001

Yamada T (2019) Thickness Constraints for Topology Optimization Using the Fictitious Physical Model. In: EngOpt 2018 Proceedings of the 6th International Conference on Engineering Optimization, Springer International Publishing, Cham, pp 483-490, DOI 10.1007/ 978-3-319-97773-7_43

Yoon GH, Jensen JS, Sigmund O (2007) Topology optimization of acoustic-structure interaction problems using a mixed finite element formulation. International Journal for Numerical Methods in Engineering 70(9):1049-1075, DOI 10.1002/nme.1900

Zhou M, Rozvany G (1991) The COC algorithm, Part II: Topological, geometrical and generalized shape optimization. Computer Methods in Applied Mechanics and Engineering 89(1-3):309-336, DOI 10.1016/0045-7825(91) 90046-9

Zhou M, Lazarov BS, Wang F, Sigmund O (2015) Minimum length scale in topology optimization by geometric constraints. Computer Methods in Applied Mechanics and Engineering 293:266-282, DOI 10.1016/j.cma.2015.05.003 